\title{
Butyrate-mediated autophagy inhibition limits cytosolic Salmonella Infantis replication in the colon of pigs treated with a mixture of Lactobacillus and Bacillus
}

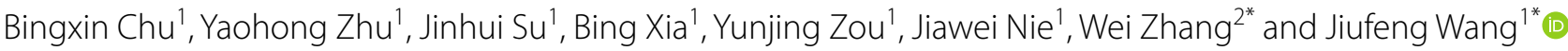

\begin{abstract}
Probiotics as an effective and safe strategy for controlling Salmonella infection are much sought after, while autophagy is a central issue in eliminating intracellular pathogens of intestinal epithelial cells. In this study, an animal model of colitis has been developed by infecting weaned pigs orally with a strain of Salmonella Infantis in order to illuminate the potential efficacy of a mixture of Lactobacillus and Bacillus (CBB-MIX) in the resistance to Salmonella infection by regulating butyrate-mediated autophagy. We found that CBB-MIX alleviated S. Infantis-induced colitis and tissue damage. Autophagy markers ATG5, Beclin-1, and the LC3-II/I ratio were significantly enhanced by S. Infantis infection, while treatment with CBB-MIX suppressed S. Infantis-induced autophagy. Additionally, S. Infantis-induced colonic microbial dysbiosis was restored by this treatment, which also preserved the abundance of the butyrateproducing bacteria and the butyrate concentration in the colon. A Caco-2 cell model of S. Infantis infection showed that butyrate had the same effect as the CBB-MIX in restraining S. Infantis-induced autophagy activation. Further, the intracellular $S$. Infantis load assay indicated that butyrate restricted the replication of cytosolic S. Infantis rather than that in Salmonella-containing vacuoles. Suppression of autophagy by knockdown of ATG5 also attenuated S. Infantisinduced cell injury. Moreover, hyper-replication of cytosolic S. Infantis in Caco-2 cells was significantly decreased when autophagy was inhibited. Our data demonstrated that Salmonella may benefit from autophagy for cytosolic replication and butyrate-mediated autophagy inhibition reduced the intracellular Salmonella load in pigs treated with a probiotic mixture of Lactobacillus and Bacillus.
\end{abstract}

Keywords: Salmonella, butyrate, autophagy, microbiota, probiotic, colon

\section{Introduction}

Salmonella enterica serovar Infantis ( $S$. Infantis), one of the most prevalent Salmonella serovars [1, 2], is of particular concern as it is a zoonotic pathogen usually transferred via contaminated food products [3-5]. S. Infantis

\footnotetext{
*Correspondence: cauzw2011@hotmail.com; jiufeng_wang@hotmail.com ${ }^{1}$ College of Veterinary Medicine, China Agricultural University, No. 2 Yuanmingyuan West Road, Beijing 100193, People's Republic of China ${ }^{2}$ Institute of Animal Husbandry and Veterinary Medicine, Beijing Academy of Agriculture and Forestry Sciences, No. 9 Shuguanghuayuan Middle Road, Beijing 100097, People's Republic of China
}

is a non-typhoidal serovar, which usually causes self-limiting inflammation of the host gut $[4,6]$. As a facultative intracellular pathogen, after invasion of epithelial cells, $S$. Infantis resides within a membrane-bound compartment referred to as the Salmonella-containing vacuole $(\mathrm{SCV})[7,8]$. Although the SCV is considered to be the intracellular niche for Salmonella, its low $\mathrm{pH}$ and nutrient-limited environment are not suitable for bacterial survival [8-10]. Recent findings indicate that Salmonella within SCV can escape into the cytoplasm where replication far exceeds that within SCV. This phenomenon is

c) The Author(s) 2020. This article is licensed under a Creative Commons Attribution 4.0 International License, which permits use, sharing, adaptation, distribution and reproduction in any medium or format, as long as you give appropriate credit to the original author(s) and the source, provide a link to the Creative Commons licence, and indicate if changes were made. The images or other third party material in this article are included in the article's Creative Commons licence, unless indicated otherwise in a credit line to the material. If material is not included in the article's Creative Commons licence and your intended use is not permitted by statutory regulation or exceeds the permitted use, you will need to obtain permission directly from the copyright holder. To view a copy of this licence, visit http://creativeco mmons.org/licenses/by/4.0/. The Creative Commons Public Domain Dedication waiver (http://creativecommons.org/publicdomain/ zero/1.0/) applies to the data made available in this article, unless otherwise stated in a credit line to the data. 
known as hyper-replication (defined as 50 bacteria per cell), a state where Salmonella expresses SPI-1 genes and synthesize flagella in preparation for further invasion $[8$, $11,12]$. These bacteria then pass through infected cells to escape into the interstitium, where they can cause infections in neighboring cells that eventually spread through the enteric cavity [12].

Autophagy is a highly conserved biological process in eukaryotes, which targets intracellular components to lysosomes for degradation $[13,14]$. Autophagy also serves as a natural immune mechanism, known as xenophagy, to eliminate bacteria that invade cells [15-17]. Paradoxically, autophagy seems to play the opposite role in the process of Salmonella infection. One earlier study showed that suppression of GTPase expression, which is necessary for autophagy, reduced Salmonella replication in HeLa cells [18]. Additional research had similar findings that knockdown of LC3 and p62 limited Salmonella proliferation [19]. Some pathogens have developed countermeasures against the cellular defense response, such as Shigella flexneri, which can restrain autophagosome generation by secreting the effector protein IcsB [20]. Furthermore, our previous study showed that Lactobacillus rhamnosus GG controlled $S$. Infantis infection by suppressing activation of intestinal epithelial cell autophagy in pigs [21]. More recently, we found that $L$. johnsonii prevented $S$. Infantis dissemination and ameliorated enteritis via suppression of autophagy in weaned pigs [22].

Gut microbiota is a critical factor in host resistance to pathogen infection. Salmonella causes intestinal inflammation and remodeling of gut microbiota, which leads to impaired colonization resistance and increases the susceptibility of the host to Salmonella [23]. Probiotics can protect the host from enteric infection by reprogramming the intestinal microbiota community and the efficacy of compound probiotics is superior to a single probiotic preparation [24, 25]. The colon has one of the most abundant microbiota of the entire intestinal tract, therefore, maintaining the stability of the host colonic microbiota is crucial for host wellbeing. Recent studies have confirmed that colonic microbiota maintains host health by producing short chain fatty acids (SCFAs) $[26,27]$. While SCFAs are absorbed by colonic mucosa, butyrate is transported preferentially and seems to be the preferred energy source for colonic cells [28]. It has been proposed that an insufficient energy supply, $70 \%$ of which normally originates from butyrate, could be a causative factor for colitis [29]. Indeed, our previous study showed that oral administration of $L$. johnsonii maintained shortchain fatty acid levels and reduced the colonization of $S$. Infantis in the intestine [30]. Remarkably, butyrate can suppress autophagy at physiological concentration [29].
However, the underlying mechanism by which gut microbiota-targeted probiotics and butyrate control $S$. Infantis infection remains to be explored.

In the present study, we showed that a probiotic mixture of Lactobacillus and Bacillus maintained butyrate concentration by regulating gut microbiota during $S$. Infantis infection. Furthermore, butyrate-mediated autophagy inhibition reduced the intracellular Salmonella load and resisted Salmonella infection in pigs treated with a probiotic mixture of Lactobacillus and Bacillus.

\section{Materials and methods \\ Bacterial strains}

Salmonella enterica serovar Infantis strain CAU1508 was isolated from the intestinal contents of weaned piglets with diarrhea in our laboratory. $S$. Infantis harboring the pFPV-mCherry or pFPV25.1 plasmid was prepared as previously described [31]. S. Infantis carrying the pFPVmCherry plasmid or pFPV25.1 plasmid can express red or green fluorescent protein, respectively. $S$. Infantis was grown in LB medium overnight with shaking at $200 \times g$ and $37{ }^{\circ} \mathrm{C}$, followed by being subcultured (1:40) into $10 \mathrm{ml}$ fresh LB medium for growth under the same conditions for 3.5-4 h. The bacteria were then centrifuged at $4000 \times g$ for $15 \mathrm{~min}$ and resuspended in saline for in vivo experiments or PBS for in vitro experiments.

Lactobacillus Johnsonii (CJ21), B. subtilis (BS15), B. licheniformis (BL21) were isolated from the intestinal contents of healthy pigs in our laboratory. L. johnsonii CJ21 was grown in De Man, Rogosa, and Sharpe (MRS) broth (Oxoid, Basingstoke, UK) for $24 \mathrm{~h}$ at $37{ }^{\circ} \mathrm{C}$ under microaerophilic conditions. B. subtilis $\mathrm{BS} 15$ and $B$. licheniformis BL21 were grown in LB medium for $24 \mathrm{~h}$ at $37^{\circ} \mathrm{C}$ under microaerophilic conditions. After overnight incubation, the bacteria were inoculated 1:100 into fresh MRS or LB broth and grown for $8 \mathrm{~h}$ until mid-log growth phase was reached.

\section{Animals and experimental design}

A total of 28 healthy pigs (Landrace $\times$ Large White) weaned at 21 days of age were all obtained from the Beijing Hog Raising and Breeding Center (Beijing, China). Piglets were selected from seven different litters, with four piglets from each litter. These four piglets were divided into four different groups. All feces were collected for Salmonella antigen detection to ensure that the piglets were not infected with Salmonella prior to the experiment. Subsequently, the piglets were fed with antibiotic-free feed and water ad libitum for 3 days to adapt to the new environment. Then piglets were used in the experiment at 25 days of age. On day 0 , pigs were randomly divided into four groups ( $\mathrm{n}=7$ per group): 
(1) control (CONT) group: intragastric administration of $10 \mathrm{~mL} /$ day sterile physiological saline for the first 7 days; (2) CBB-MIX group: intragastric administration of $10 \mathrm{~mL} /$ day CBB-MIX: L. johnsonii CJ21 $1 \times 10^{9} \mathrm{CFU} /$ $\mathrm{mL})$, B. subtilis BS15 $\left(1 \times 10^{6} \mathrm{CFU} / \mathrm{mL}\right)$, B. licheniformis BL21 $\left(1 \times 10^{6} \mathrm{CFU} / \mathrm{mL}\right)$ for the first 7 days; (3) SI group: intragastric administration of $10 \mathrm{~mL} /$ day physiological saline for the first 7 days and challenged with $S$. Infantis $\left(5 \times 10^{10} \mathrm{CFU} / \mathrm{mL}, 10 \mathrm{~mL} /\right.$ day) on day 8; (4) CBBMIX + SI group: intragastric administration of $10 \mathrm{~mL} /$ day CBB-MIX solution for the first 7 days and challenged with $S$. Infantis $\left(5 \times 10^{10} \mathrm{CFU} / \mathrm{mL}, 10 \mathrm{~mL}\right)$ on day 8 . On day 10, one piglet from each group was slaughtered to determine whether the experimental model for colon damage caused by Salmonella Infantis was successfully established. Finally, on day 13, all the pigs were euthanized and tissue samples were immediately collected.

\section{Reagents and antibodies}

Lyso-Tracker Red (L8010), 4',6'-diamidino-2-phenylindole (DAPI) solution (C0060), and Calcein-AM/ PI (CA1630) were purchased from Beijing Solarbio Science \& Technology Co., Ltd. (Beijing China). Chlorquine diphosphate salt (C6628), butyrate sodium (B5887), and Triton X-100 (T8787) were obtained from Sigma-Aldrich (St. Louis, USA). Dulbecco's Modified Eagle Medium (DMEM)/High Glucose (SH30022.01) and phosphate buffered saline (PBS) were purchased from GE Healthcare Life Sciences HyClone Laboratories (Utah, USA). Fetal bovine serum (FBS) was obtained from Thermo Fisher Scientific (Rockford, USA). Lipofectamine ${ }^{\text {TM }}$ RNAiMAX transfection reagent (13778075) was obtained from ThermoFisher Scientific (Rockford, USA). Conventional chemosynthetic siRNA for ATG5 was obtained from Living Biotechnologies Co., Ltd. The following primary antibodies: rabbit anti-ATG5 polyclonal antibody (10181-2-AP); rabbit anti-Beclin 1 polyclonal antibody (11306-1-AP); rabbit anti-p62/SQSTM1 polyclonal antibody (18420-1-AP); rabbit anti-LAMP 1 polyclonal antibody (21997-1-AP); mouse anti-LAMP 2 polyclonal antibody (66301-1-lg); rabbit anti-ATP6V1A polyclonal antibody (17115-1-AP); rabbit anti-ATP6V1B2 polyclonal antibody (15097-1-AP); rabbit anti-ATP6V1E1 polyclonal antibody (15280-1-AP); rabbit anti-Occludin polyclonal antibody (27260-1-AP); mouse anti-GAPDH monoclonal antibody (60004-1-lg); and mouse anti-Beta ACTIN monoclonal antibody (60008-1-lg) were purchased from Proteintech Group Inc (Rosemont, IL 60018, USA). Rabbit anti-LC3A/B polyclonal antibody (\#4108) was obtained from Cell Signaling Technology (Danvers, MA 01923, USA). Rabbit anti-ZO-1 polyclonal antibody was purchased from ThermoFisher Scientific (Rockford, USA). Rabbit anti-Claudin-1 polyclonal antibody (ab15098) was obtained from abcam (Cambridge, UK). The secondary antibodies used for Western Blotting were purchased from Proteintech Group Inc: HRP-conjugated Affinipure Goat Anti-Rabbit IgG(H+L) (SA00001-2) and HRP-conjugated Affinipure Goat Anti-Mouse IgG(H+L) (SA00001-1). Alexa Fluor 488-labeled Goat Anti-Rabbit $\lg \mathrm{G}(\mathrm{H}+\mathrm{L})$ and Alexa Fluor 555-labeled Donkey AntiRabbit $\lg \mathrm{G}(\mathrm{H}+\mathrm{L})$ were obtained from Beyotime Biotechnology (Shanghai, China).

\section{Histopathologic scoring}

To assess intestinal pathology, mid-segments $(10 \times 15 \times 3 \mathrm{~mm})$ of the colon were excised, rinsed with saline and then immersed in $4 \%$ paraformaldehyde. Paraffin-embedded samples of the colon tissues were sectioned $(3 \mu \mathrm{m})$ and stained with hematoxylin and eosin. The level intestinal inflammation was scored as previously described [32].

\section{Western blotting}

Total proteins were extracted from the middle segment of the colon or each well of Caco-2 cells using RIPA buffer (Solarbio, Beijing, China) containing a protease/phosphatase inhibitor cocktail (Cell Signaling Technology, USA). Protein concentration was quantified using a BCA Protein Assay kit (23227, ThermoFisher Scientific), prior to separation by sodium dodecyl sulfate-polyacrylamide gel electrophoresis and transfer onto polyvinylidene fluoride membranes. After blocking the membranes with 5\% skim milk at room temperature for $1.5 \mathrm{~h}$ and incubation at $4{ }^{\circ} \mathrm{C}$ overnight, different membranes were incubated with the following corresponding primary antibodies: anti-ATG5 (1:500), anti-Beclin 1 (1:1000), anti-p62/ SQSTM1 (1:2000), anti-LAMP 1 (1:2000), anti-LAMP 2 (1:1000), anti-ATP6V1A (1:1000), anti-ATP6V1B2 (1:2000), anti-ATP6V1E1 (1:2000), anti-occludin (1:1500), anti-GAPDH (1:5000), anti-beta ACTIN (1:5000), antiLC3A/B (1:1000), anti-ZO-1 (1:250) and anti-claudin-1 (1:2000). Membranes were then subjected to horseradish peroxidase-conjugated secondary antibodies (1:5000) raised against the corresponding species source for the primary antibody for $1 \mathrm{~h}$ at room temperature. Then ECL western blotting substrate (Tanon Science \& Technology Co., Ltd. Shanghai, China) was placed on the membranes. The images were obtained with a Tanon 6200 chemiluminescence imaging workstation (Tanon Science \& Technology Co., Ltd. Shanghai, China). Each test was performed in triplicate.

\section{Immunofluorescence}

Colonic samples were fixed in $4 \%$ formaldehyde for $24 \mathrm{~h}$ and embedded in paraffin prior to sectioning. The sections were dewaxed, rehydrated, and subjected to 
microwave antigen retrieval. Caco- 2 cells were treated as described above. Samples were fixed with $4 \%$ paraformaldehyde for $7 \mathrm{~min}$ and permeabilized with PBS for $13 \mathrm{~min}$. In the following step, all the tissue and cell samples were blocked with $2 \%$ bovine serum albumin for $1.5 \mathrm{~h}$ at room temperature. Then samples were incubated with anti-LAMP1 antibody (1:100), anti-ZO-1 antibody (1:50), anti-claudin-1 antibody (1:1000), and anti-occludin antibody $(1: 200)$ at $4{ }^{\circ} \mathrm{C}$ overnight. After washing three times with PBS, samples were incubated with Alexa Fluor 488 goat anti-rabbit or Alexa Fluor 555 donkey anti-rabbit secondary antibodies at room temperature for $1 \mathrm{~h}$. DAPI was used to stain the nuclei for $10 \mathrm{~min}$ at room temperature. Images were obtained on a Nikon A1 confocal laser scanning microscope. At least 100 infected cells were counted for each independent experiment.

\section{Microbial genomic DNA extraction and 16S rRNA gene amplification}

Samples of colonic contents (from middle segment of the colon) were collected immediately after sacrifice. Total microbial genomic DNA was extracted using a QIAamp DNA Microbiome kit (Qiagen, Hilden, Germany) according to the manufacturer's instructions and the purified products checked on a $1 \%$ agarose gel, prior to the DNA concentration and purity being determined with a NanoDrop 2000 UV-vis spectrophotometer (Thermo Scientific, Wilmington, USA).

The hypervariable region $\mathrm{V} 3-\mathrm{V} 4$ of the bacterial $16 \mathrm{~S}$ rRNA gene was amplified with primer pairs 338F (5'ACTCCTACGGGAGGCAGCAG-3') and 806R (5'-GGA CTACHVGGGTWTCTAAT-3') in an ABI GeneAmp 9700 PCR thermocycler (Applied Biosystems, CA, USA). The PCR amplification procedure was performed as follows: initial denaturation at $95{ }^{\circ} \mathrm{C}$ for $3 \mathrm{~min}$, followed by 30 cycles of denaturing at $95^{\circ} \mathrm{C}$ for $30 \mathrm{~s}$, annealing at $55{ }^{\circ} \mathrm{C}$ for $30 \mathrm{~s}$ and extension at $72{ }^{\circ} \mathrm{C}$ for $45 \mathrm{~s}$, and single extension at $72{ }^{\circ} \mathrm{C}$ for $10 \mathrm{~min}$, and subsequently kept at $10{ }^{\circ} \mathrm{C}$. The PCR mixtures contained $4 \mu \mathrm{L} 5 \times$ TransStart FastPfu buffer, $2 \mu \mathrm{L}$ dNTPs $(2.5 \mathrm{mM}), 0.8 \mu \mathrm{L}$ forward primer $(5 \mu \mathrm{M}), 0.8 \mu \mathrm{L}$ reverse primer $(5 \mu \mathrm{M}), 0.4 \mu \mathrm{L}$ TransStart FastPfu DNA Polymerase, 10 ng of template DNA, and lastly $\mathrm{ddH}_{2} \mathrm{O}$ to a final volume of $20 \mu \mathrm{L}$. PCR reactions were performed in triplicate and then the three PCR products were mixed for the electrophoresis on a $2 \%$ agarose gel. The specific 468 -bp PCR products were extracted and purified using an AxyPrep DNA Gel Extraction kit (Axygen Biosciences, Union City, USA), and quantified using a Quantus ${ }^{\mathrm{TM}}$ Fluorometer (Promega, Madison, USA) according to the manufacturer's instructions, prior to sequencing as described below.

\section{S rRNA gene sequencing and bioinformatics analysis}

Purified 16S rRNA gene amplicons were pooled in equimolar concentrations and paired-end sequenced $(2 \times 300 \mathrm{bp})$ on an Illumina MiSeq platform (Illumina, San Diego, USA) according to the standard protocols provided by Majorbio Bio-Pharm Technology Co. Ltd. (Shanghai, China). The raw 16S rRNA gene sequencing reads were demultiplexed, quality-filtered using Trimmomatic and merged by FLASH with the following criteria: (i) the 300 bp reads were truncated at any site receiving an average quality score of $<20$ over a 50 bp sliding window, and truncated reads shorter than $50 \mathrm{bp}$ and reads containing ambiguous characters were also discarded; (ii) only overlapping sequences longer than $10 \mathrm{bp}$ were assembled according to their overlapped sequence, where the maximum mismatch ratio of the overlap region was 0.2 and reads that could not be assembled were discarded; and (iii) samples were distinguished according to the barcode and primer sequences, and the sequence direction was adjusted. Note that exact barcode matching and a maximum of two nucleotide mismatches in the primer sequences were required for use. Operational taxonomic units (OTUs) with 97\% similarity cutoff were clustered using UPARSE (version 7.1, http://drive5.com/ uparse/), and chimeric sequences were identified and removed. The taxonomy of each OTU representative sequence was analyzed by the Ribosomal Database Project (RDP) Classifier (http://rdp.cme.msu.edu/) against the Silva SSU128 database using a confidence threshold of $70 \%$. The alpha diversity (Shannon and Simpson), richness (ACE and Chao1), rarefaction curve, and Venn diagram analysis were performed using Mothur version 1.31.2 (http://www.mothur.org). A heatmap was created based on the relative abundance of the OTUs using $R$ software (version 2.15) (http://www.R-project.org). Multivariate data analysis, including partial least squaresdiscriminate analysis (PLS-DA) and principle coordinate analysis (PCoA) based on unweighted unifrac distance, was performed using Simca-P 12.0 (Umetrics, Umea, Sweden) and R (version 3.2.1), respectively.

\section{Quantification of SCFAs}

The concentrations of acetate, propionate, butyrate, isobutyrate, isovalerate, and valerate in the colonic contents were determined using gas chromatography (Agilent 7890 A, Agilent Technologies, Santa Clara, USA) as previously described [33].

\section{Cell culture and infection}

$1 \times 10^{6}$ cells (per well) were seeded in 6-well culture plates, and $1 \times 10^{5}$ cells (per well) were seeded in 24-well culture plates. Caco-2 cells were cultured in DMEM/ 
High Glucose media supplemented with $10 \%$ FBS and $1 \%$ penicillin streptomycin at $37{ }^{\circ} \mathrm{C}$ in a $5 \% \mathrm{CO}_{2}$ incubator. The butyrate was diluted to $10 \mathrm{mM}$ in DMEM medium. The cells were incubated with $10 \mathrm{mM}$ butyrate for $2 \mathrm{~h}$ and then washed three times with PBS to remove the residual butyrate. $S$. Infantis was diluted in medium and added at a multiplicity of infection (MOI) of 50 . After $30 \mathrm{~min}$, the cells were washed three times with PBS to remove non-internalized bacteria. Subsequently, the cells were cultured in fresh growth medium containing $100 \mathrm{mg} / \mathrm{mL}$ gentamicin for $2 \mathrm{~h}$ to kill any remaining extracellular $S$. Infantis, followed by replacing this with growth medium containing $10 \mathrm{mg} / \mathrm{mL}$ gentamicin to restrict the extracellular growth of $S$. Infantis for the remaining incubation time until sample collection. Butyrate was not added to the medium during the infection, however, after the medium was replaced post-infection, all reagents contained $10 \mathrm{mM}$ butyrate. Three replicates were conducted for each cell sample.

\section{Scanning electron microscopy (SEM)}

Caco- 2 cells were collected at the designed time points and fixed with $3 \%$ glutaraldehyde for $48 \mathrm{~h}$. The samples were treated using a standard SEM procedure, as previously described [34].

\section{Chloroquine (CQ) resistance assay}

A CQ resistance assay was performed to obtain the quantity of cytosolic bacteria as a fraction of the total bacteria internalized into cells, as previously described $[8,11]$. Cells were co-incubated with $700 \mu \mathrm{M}$ CQ and $100 \mu \mathrm{g} /$ $\mathrm{mL}$ gentamicin simultaneously for $1 \mathrm{~h}$ to quantify the number of cytosolic $S$. Infantis, while control cells were incubated with gentamicin only. At $0,2,4,6,8,10$, and $12 \mathrm{hpi}$, cells were washed three times with PBS and lysed in $200 \mu \mathrm{L}$ PBS containing $0.3 \%$ Triton X-100. Bacteria released from cells were serial diluted and plated on LB agar plates (with $100 \mu \mathrm{g} / \mathrm{mL}$ ampicillin to select $S$. Infantis with pFPV-mCherry plasmid) to quantify the bacteria. Three independent replicates were conducted for each timepoint.

\section{Calcein-AM/PI cell mortality assay}

Cells were seeded into 24-well plates in complete medium. At $8 \mathrm{hpi}$, all media was removed and $2 \mathrm{ml}$ of $1 \times$ AssayBuffer was added to each well. The cell mortality was evaluated by double staining: $2 \mu \mathrm{M}$ calcein-AM and $4.5 \mu \mathrm{M}$ PI for $15 \mathrm{~min}$ at $37^{\circ} \mathrm{C}$. The viable and dead cells (yellow green fluorescence and red fluorescence respectively) were detected simultaneously with a $490 \pm 10 \mathrm{~nm}$ excitation filter under a fluorescence microscope. Five visual fields were randomly selected and 100 cells were counted from each view. Finally, the percentage of cells with each color was calculated.

\section{RNA interference}

Caco- 2 cells were seeded in 24 well plates and grown to 60-80\% confluence at the time of transfection. Cells were transfected with Atg5-siRNA diluted with the RNAiMAX transfection reagent according to the manufacturer's instructions. At $5 \mathrm{~h}$ after transfection, the media was removed and cells were incubated with DMEM at $37^{\circ} \mathrm{C}$ for $48 \mathrm{~h}$.

\section{Data deposition and data bank accession number}

The raw 16S rRNA gene sequences have been deposited into the US National Center for Biotechnology Information Sequence Read Archive database (accession number: PRJNA597824).

\section{Statistical analysis}

Experiments were conducted at least three times. The statistical analysis was performed using GraphPad Prism 7 with a one-way ANOVA or a t-test with Bonferroni correction. Data were presented as mean $\pm S E M$ and a $P$-value $<0.05$ was regarded as statistically significant. For data of relative abundance of taxa at the phylum, class, family, genus, and species levels from 16S rRNA gene sequencing, differences between the least-square means were compared using Tukey's post hoc test with multiple testing corrections applied according to the BenjaminiHochberg's false discovery rate ( $q$ value), based on the global $\mathrm{P}$ values of the variables compared. A $P$-value of $<0.05$ with a $q$ value of $<0.05$ was considered indicative of significance.

\section{Results \\ Oral CBB-MIX alleviated the S. Infantis-induced colon injury in weaned pigs}

To investigate whether the CBB-MIX plays a protective role against $S$. Infantis infection, we conducted a comprehensive histological analysis of the colon. First, histologic analysis showed that, compared with the CONT group, pigs in the SI group exhibited obvious pathological injury in the colon in the form of epithelial stripping and fissures; mucosa hyperemia; inflammatory cell infiltration; submucosal connective tissue loosening and hyperemia (Figure 1A). However, oral administration of CBB-MIX relieved the severity of $S$. Infantis-induced histopathological injury in the colon. While increased intestinal inflammation compared with the colon of CONT pigs $(P<0.001$, Figure $1 \mathrm{~B})$ was observed in the SI group, lower histologic scores were observed in the colon of CBB-MIX + SI pigs compared with that of SI pigs $(P=0.01)$. The intestinal mucosal 


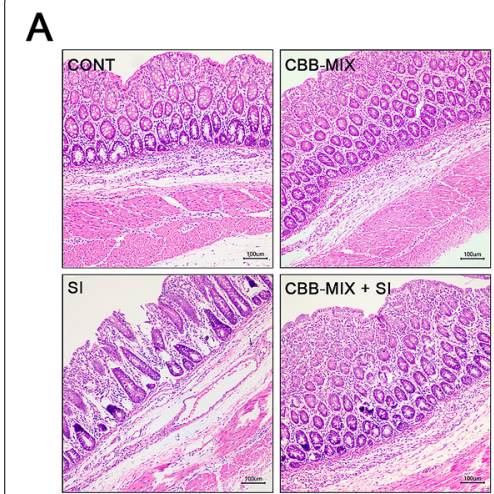

B

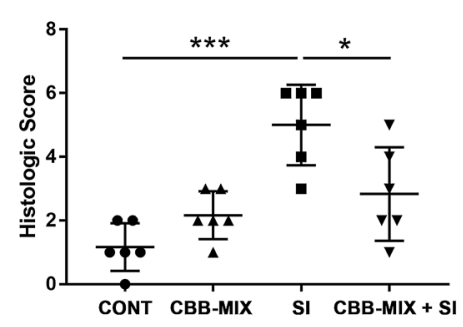

C
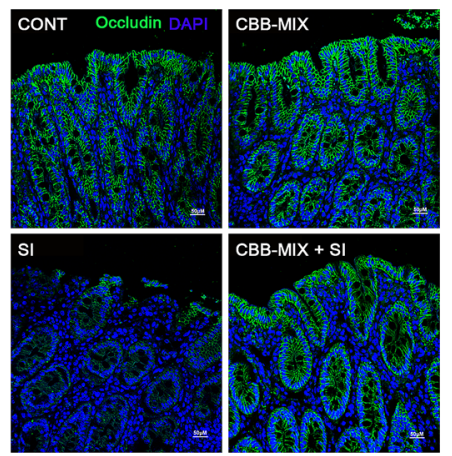

$\mathrm{D}$

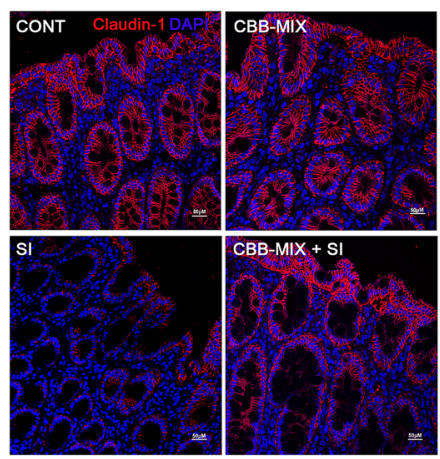

$\mathrm{E}$
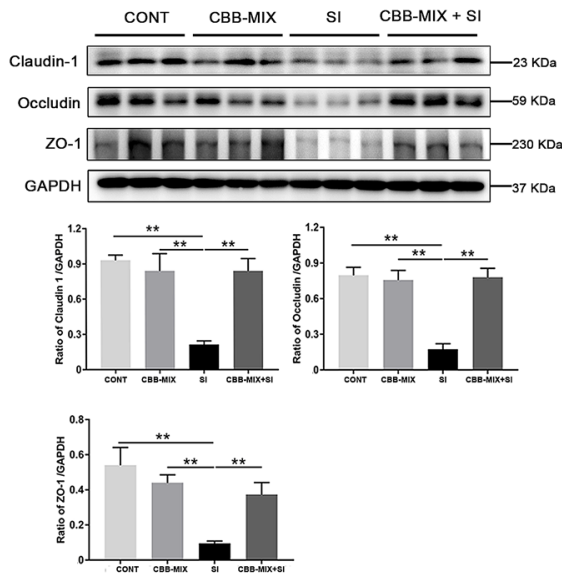

Figure 1 Orally fed CBB-MIX alleviated the colon injury of weaned pigs caused by S. Infantis. A, B Colon histological scores and representative photomicrographs of H\&E stained section. C, D Detection of the distribution and expression of occludin (Green) and claudin-1 (Red) in colon tissue by immunofluorescence microscopy. Blue: DAPI. Scale bar, $50 \mu \mathrm{m}$. E, Western blot analysis for the expression levels of claudin-1, occludin and ZO-1 proteins in colon tissues from piglets. The lower panel shows the quantitation analysis by Image J software. All these data are presented as mean $\pm \operatorname{SEM}(n=6),{ }^{*} P<0.05,{ }^{* *} P<0.01,{ }^{* * *} P<0.001$

mechanical barrier is an important physiological barrier against invasion of pathogenic microorganisms $[35,36]$. Immunofluorescence results indicated that $S$. Infantis challenge disrupted the tight junction structure of the epithelium and resulted in the fracture and disappearance of intercellular occludin (Figure 1C) and claudin-1 (Figure 1D) proteins. Oral administration of the CBB-MIX preserved the complete tissue structure and maintained the uniform distribution of intercellular occludin and claudin-1 during $S$. Infantis infection. Western blot analysis also demonstrated that compared with the CONT group, lower levels of claudin-1, occludin and ZO-1 proteins were observed in the SI group $(P=0.002, P=0.001$ and $P=0.002$, respectively, Figure 1E). Conversely, higher levels of claudin-1, occludin and ZO-1 proteins were found in the CBB-MIX + SI group compared with the SI group $(P=0.002, P=0.001$ and $P=0.003$, respectively, Figure $1 \mathrm{E})$. These results indicated that oral administration of the probiotic CBB-MIX attenuated $S$. Infantis-induced colon injury in pigs.

\section{Orally fed CBB-MIX affected the colonic microbiota} composition and maintained the abundance of butyrate-producing bacteria during $S$. Infantis infection High throughput 16S rRNA gene sequencing was performed to explore the effect of CBB-MIX on the colonic microbial community during $S$. Infantis infection. After optimizing the raw sequences, a total of $1,224,788$ sequences from 24 samples were obtained for further analysis. The slopes of the rarefaction curves based on the Sobs and Shannon indices tended to be gentle (Figure $2 \mathrm{~A}$ ), indicating that the sequencing data had reached saturation and the sequencing depth reflected the vast majority of microbial diversity information. As shown in the rank-abundance curves (Figure 2A), a small number of OTUs dominated and the majority of OTUs were present at low abundance in the colonic microbiota. The rarefaction curves based on the Sobs, Shannon and rank abundance curves showed that the CONT, CBB-MIX, and CBB-MIX + SI groups had greater taxon richness than the SI group. One-way ANOVA tests showed that compared with the CONT group, a decreased number 
of OUT were observed (as shown by the Sobs and Shannon indices) in the SI group, but not in pigs from the CBB-MIX and CBB-MIX + SI groups (Figure 2B). Compared with the SI group, pigs in the CBB-MIX and CBBMIX + SI groups had a higher number of OTU. The PCoA based on unweighted unifrac distance showed that the coordinate points in the CBB-MIX group were arranged closer and shorter than those in the other three groups, indicating the CBB-MIX could regulate the consistency of the colonic microbiota structure. The colonic microbiota of SI pigs was clearly distinguishable from the other three groups, and oral CBB-MIX shifted the overall structure of the $S$. Infantis-disrupted colonic microbiota toward that of the CONT and CBB-MIX pigs (Figure 2C). Additionally, the PLS-DA based on the unweighted unifrac distance also displayed similar results (Figure 2C), demonstrating that treatment with the CBB-MIX maintained the stability and diversity of colonic microbiota under disturbance by $S$. Infantis.

In total, 15 phyla were identified across all the samples (Figure 2D). The results of heat map indicated that the dominant phyla were the same in the different samples at the phylum level, with Firmicutes and Bacteroidetes accounting for the majority of the total sequences (Figure 2D). Compared with the CONT group, S. Infantis infection increased the abundance of Bacteroidetes $(P=0.034)$, but this increase was inhibited by treatment with the CBB-MIX $(P=0.046)$ (Figure 2E). Heat map analysis revealed the top 50 genera with relative abundance at the genus level (Figure 2F). Compared with the CONT group, there was an obvious shift in the colonic microbiota in the SI group, while the microbiota profiles in CBB-MIX and CBB-MIX + SI groups were similar to that in the CONT group (Figure 2F). The ANOVA results showed that compared with the CONT group, the genera Prevotella_9 $(P=0.002)$, Clostridium_sensu_stricto_1 $(P=0.004)$, unclassified_f _Peptostreptococcaceae $(P=0.010)$, and Terrisporobacter $(P=0.039)$ were markedly enriched in the SI group, whereas oral administration of CBB-MIX prevented this increase. Indeed, this treatment reversed the $S$. Infantis-induced decrease in the genera Lactobacillus $(P=0.039)$, Blautia $(P=0.016)$, unclassified_f_Lac hnospiraceae $(P=0.049)$, Faecalibacterium $(P=0.027)$, Parabacteroides $(P=0.017)$, Ruminococcaceae_UCG$002(P=0.024)$, norank_f_Bacteroidales_S24-7_group $(P=0.013)$, Roseburia $(P=0.041)$, Phascolarctobacterium $(P=0.035)$, Coprococcus_3 $(P=0.026$, Figure $2 \mathrm{G})$. Of particular note was that the oral CBB-MIX treatment maintained the abundance of butyrate-producing bacteria, including Lactobacillus, Blautia, Ruminococcaceae UCG-002, Faecalibacterium, and populations belonging to Lachnospiraceae.

\section{Effect of oral CBB-MIX on the level of SCFAs during $S$. Infantis infection}

Next, the levels of each of five SCFAs were detected in the colon contents of pigs in response to $S$. Infantis infection alone or after CBB-MIX pretreatment. Acetate and propionate were found to be elevated in the CBBMIX and CBB-MIX + SI groups, compared with the SI group (Figure 3A, B). When compared with the CONT group, $S$. Infantis infection markedly decreased the level of butyrate in the colon $(P=0.020$, Figure $3 C)$. This decrease in butyrate level could be significantly reversed by pretreatment with CBB-MIX ( $P=0.0007$, Figure $3 C)$. There was no difference in the level of valerate among all groups (Figure 3D). Isobutyrate and isovalerate concentrations were lower in the CBB-MIX group than those of the CONT group $(P=0.0003$ and $P=0.006$, Figure $3 \mathrm{E}$, F). Moreover, compared with the CONT group, $S$. Infantis infection resulted in a marked decrease in the level of total SCFAs in the colon $(P=0.049)$, while this decrease was reversed by oral administration of CBB-MIX $(P=0.0005$, Figure 3G).

\section{Butyrate attenuated cell damage by limiting the replication of cytosolic $S$. Infantis in Caco-2 cells}

The effect of butyrate on $S$. Infantis infection was explored in Caco-2 cells. Salmonella can proliferate within intestinal epithelial cells, and eventually a massive number of Salmonella escape from the infected cells into the intestinal cavity [12]. SEM images showed that $S$. Infantis punched holes in the outer membranes of Caco-2 cells and extruded toward the apical side, causing massive leakage of the cellular contents (Figure 4A). Immunoblot assays indicated that $S$. Infantis decreased occludin, claudin-1, and ZO-1 protein levels at $8 \mathrm{hpi}$ compared with non-infected cells, while butyrate treatment preserved the high expression of these three tight junction proteins during $S$. Infantis infection (Figure 4B). Immunofluorescence assays further confirmed that $S$. Infantis caused the reduction of ZO-1 fluorescence area, and induced a disordered arrangement and aggregation of ZO-1 between cells, while butyrate reversed these phenomena (Figure 4C). The calcein-AM/PI cell mortality assay showed that compared with control cells, $S$. Infantis infection increased cell mortality by $34.3 \%$, whereas coincubation with butyrate lowered by this by $7.5 \%$ (Figure 4D). These results indicated that butyrate attenuated $S$. Infantis-induced cell damage.

We deduced that butyrate might limit the replication of intracellular $S$. Infantis, since the number of intracellular bacteria in the Butyrate + SI group was obviously less than that of the SI group (Figure 4C). Then, we quantified intracellular bacteria to explore whether butyrate reduced the replication of Salmonella, and 


\section{A}

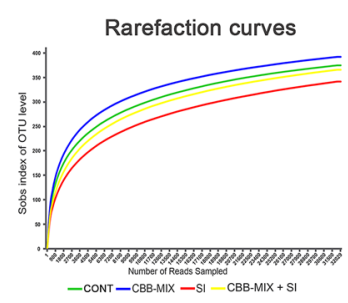

B

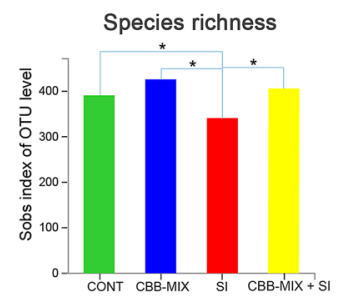

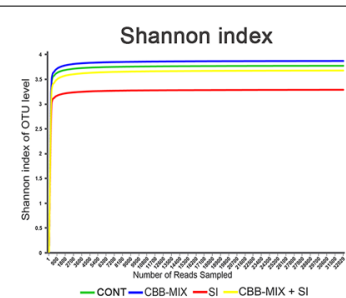
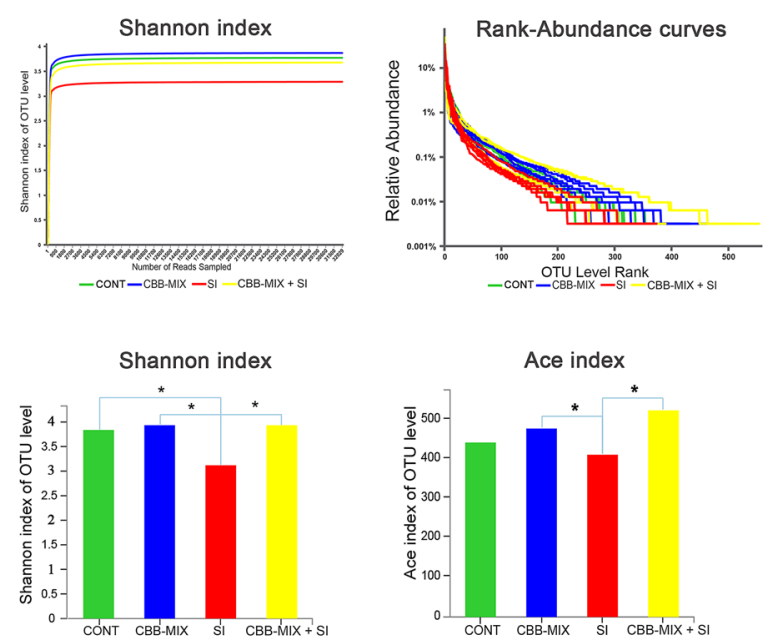

C
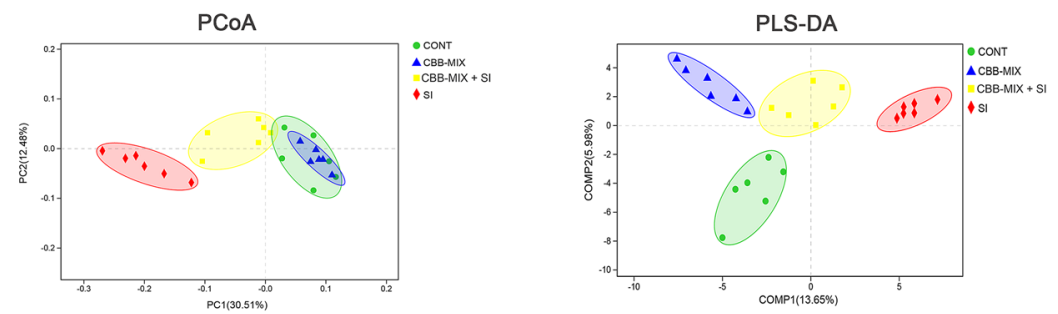

D

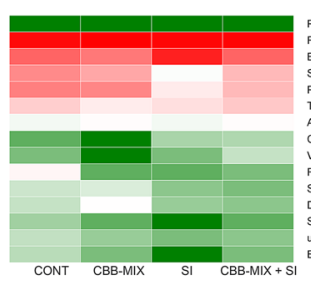

F

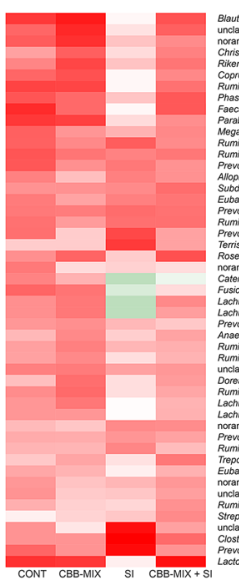

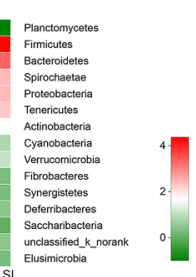

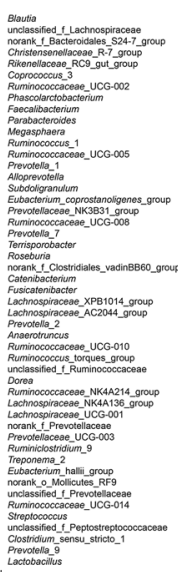

E

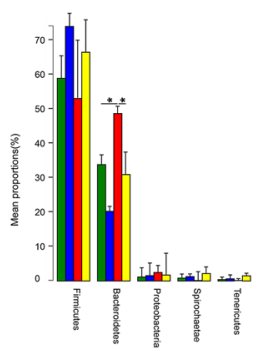

G

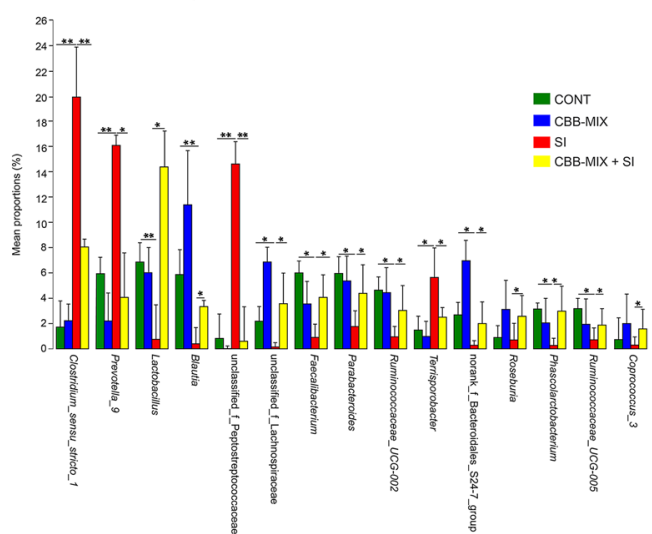

Figure 2 Diversity analysis of colonic microbiota. A Diversity analysis of colonic microbiota. The rarefaction abundance curves, Shannon index, and rank abundance curves were used to assess the colonic microflora diversity at $97 \%$ similarity in 4 groups $(n=6)$. B Difference analysis of alpha diversity index (Sobs, Shannon, and Ace index) between groups. Alpha diversity was illustrated by number of observed OTU using a one-way ANOVA. * $P<0.05$. C Comparative analysis of colonic microbiota. Beta diversity was illustrated by Principal coordinate analysis (PCOA) of the overall microbial composition (Distance Algorithm: unweighted unifrac, ANOSIM R: 0.591, $P=0.001$ ). Partial Least Squares Discriminant Analysis (PLS-DA) was used to represent sample grouping analysis results (Distance Algorithm: unweighted unifrac). D Heatmap analysis showed the relative abundance of the bacterial at the phylum level in four groups. $\mathbf{E}$ Differences in the relative abundance of genera from the 4 groups are shown using one-way ANOVA. ${ }^{*} P<0.05,{ }^{*} P<0.01,{ }^{* * *} P<0.001$. F Heatmap analysis showed the relative abundance of the top 50 genera in four groups. $\mathbf{G}$ Differences in relative abundance of genera from the $4 \mathrm{groups}$ are shown using one-way ANOVA. All these data were presented as mean \pm SEM. ${ }^{*} P<0.05,{ }^{*} P<0.01$. 


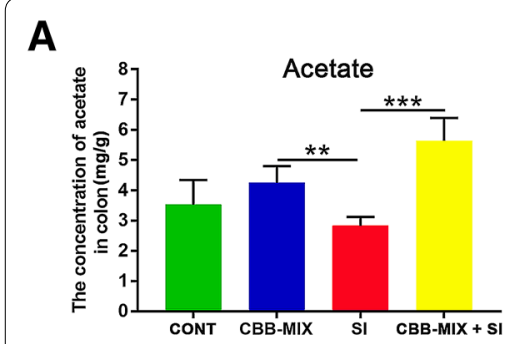

B

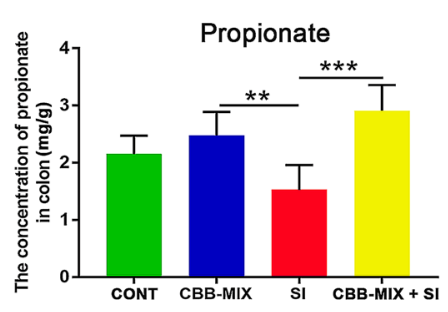

E

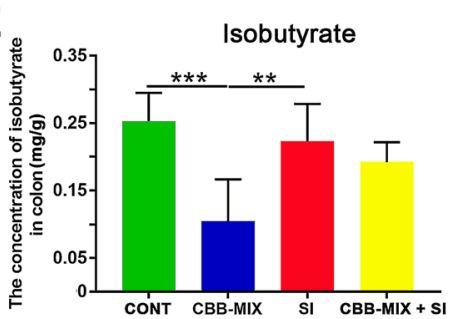

C

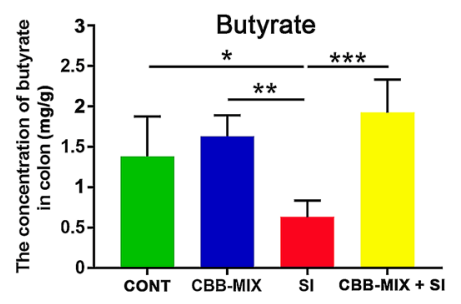

$\mathbf{F}$

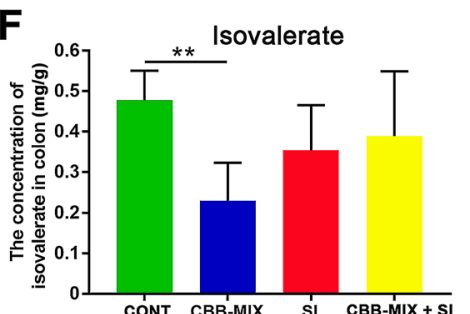

G

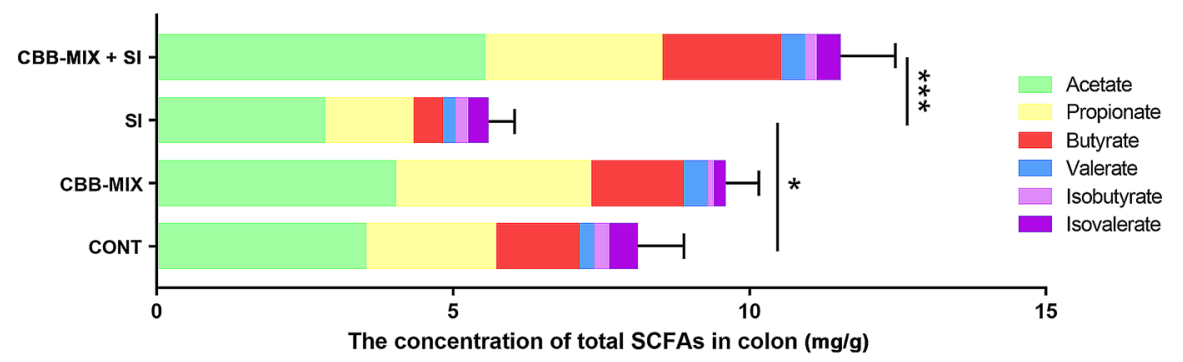

Figure 3 The effect of oral administration of CBB-MIX on the level of SCFAs during S. Infantis infection. A-G Concentrations of acetate, propionate, butyrate, valerate, isobutyrate, isovalerate, and total SCFAs levels in the colonic contents of pigs. The data were presented as mean $\pm \operatorname{SEM}(\mathrm{n}=6),{ }^{*} P<0.05,{ }^{*} P<0.01 .{ }^{* *} P<0.001$.

adopted a gentamicin protection assay to define the percentage of infected cells containing different numbers of bacteria per cell. The results showed that butyrate did not attenuate the invasiveness of $S$. Infantis during the first 2 hpi (Figure 4E). Nevertheless, the replication of intracellular $S$. Infantis was restricted by butyrate between 6 to $12 \mathrm{hpi}$ (Figure 4E). There are two distinct subpopulations of intracellular Salmonella in epithelial cells: cytosolic or SCV. Cytosolic Salmonella replicates to much higher than do SCV bacteria, a phenomenon known as hyper-replication [37, 38]. This suggested that butyrate might have specifically restricted this hyperreplication of cytosolic Salmonella. The chloroquine (CHQ) resistance assay determined that the quantity of cytosolic Salmonella comprise the majority of the total amount of intracellular bacteria at $6 \mathrm{hpi}$ (Figure 4F). In the SI group, approximately $37 \%$ of total infected cells contained hyper-replicating bacteria, as recognized by the high numbers ( $>50$ bacteria per cell) of intracellular bacteria (Figure 4G). However, we found that in the
Butyrate + SI group only $3 \%$ of infected cells contained hyper-replicating bacteria and $86 \%$ of infected cells contained less than 20 bacteria per cell (Figure 4G). We then distinguished these two subpopulations by examining the colocalization of these bacteria with LAMP1, a marker protein for the SCV membrane. These data showed that butyrate treatment did not change the quantity of SCV Salmonella, but that the cytosolic Salmonella quantity decreased significantly (Figure $4 \mathrm{H}$ ). Collectively, the results suggested that butyrate limited the replication of cytosolic $S$. Infantis in Caco-2 cells.

\section{Butyrate suppressed S. Infantis-induced autophagy} activation and alleviated lysosomal damage caused by $S$. Infantis in Caco-2 cells

Autophagy can serve as a natural defense mechanism to restrict or eliminate intracellular bacteria [21, 26, 30]. We next investigated the effects of $S$. Infantis on autophagy. Compared with the CONT group, $S$. Infantis infection increased the levels of ATG5, and Beclin1 proteins and 


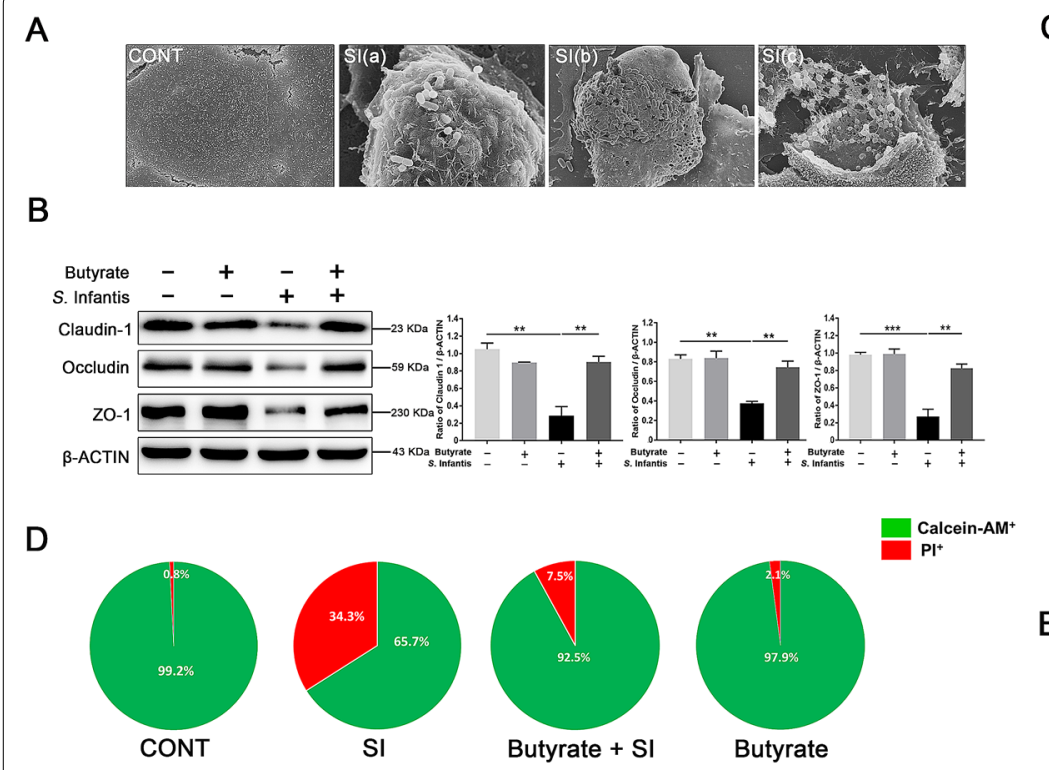

F

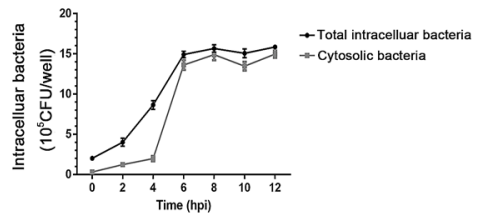

G

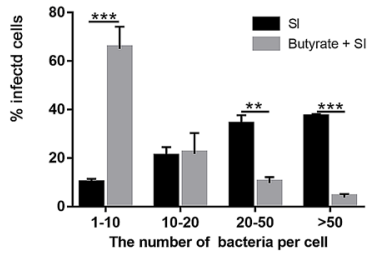

$\mathrm{H}$
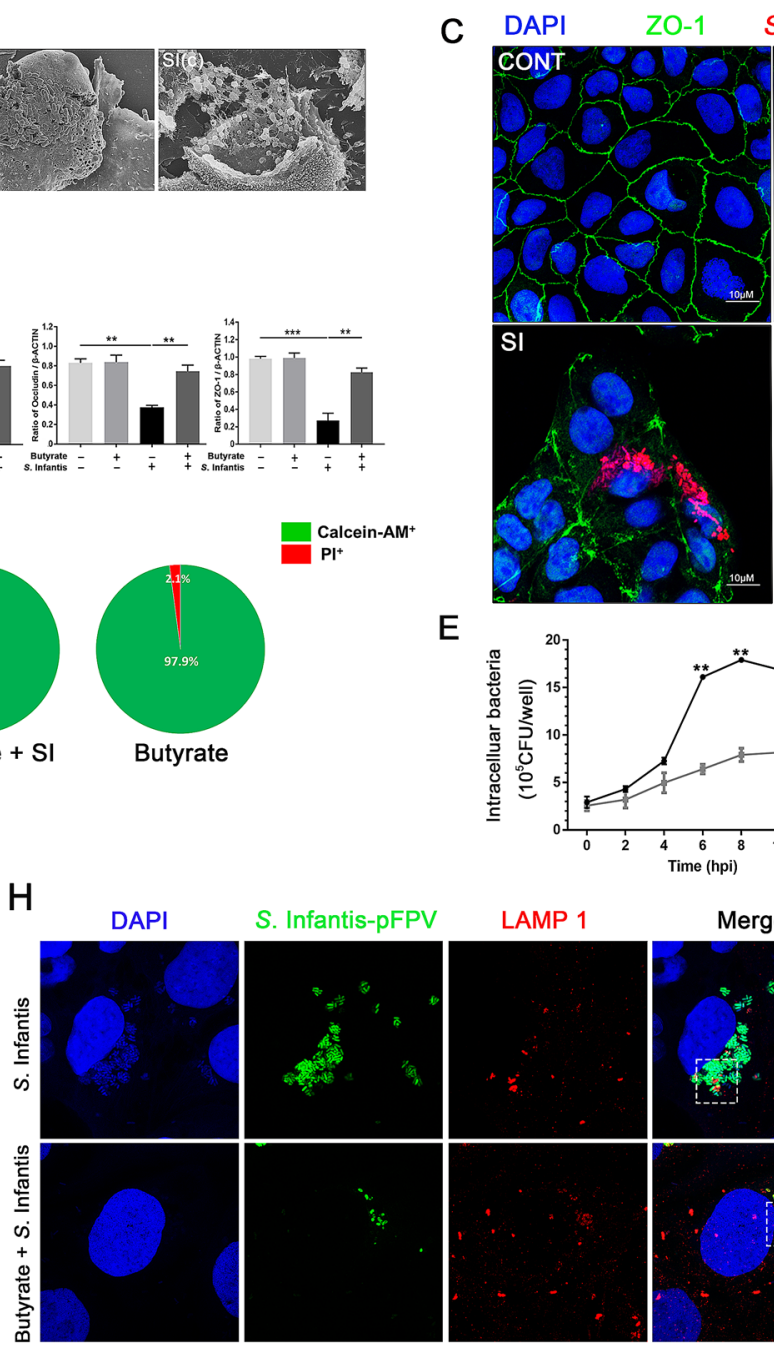

S. Infantis-mcherry

SI
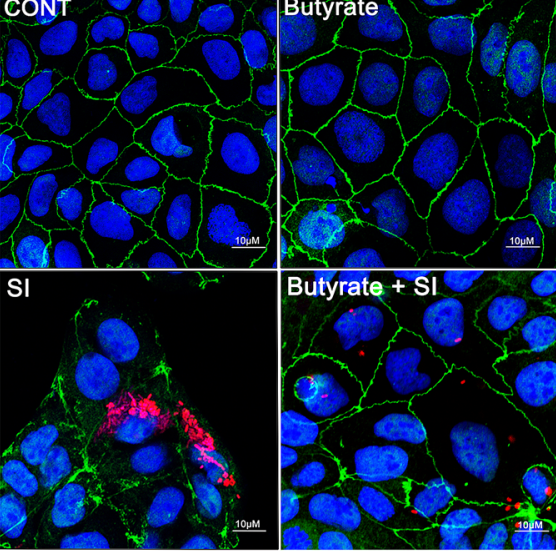

$\mathrm{E}$

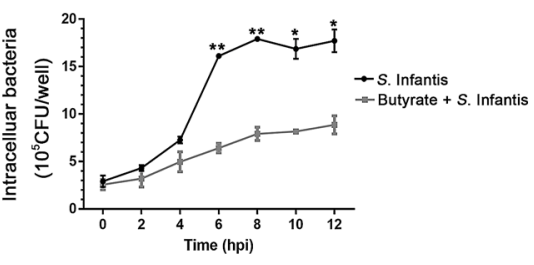

LAMP 1
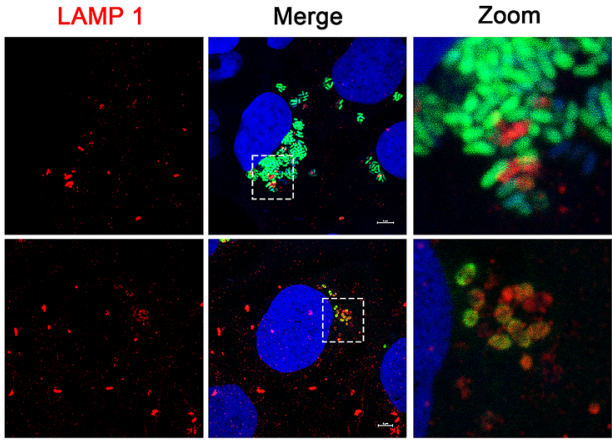

Figure 4 Butyrate reduced cell damage by limiting replication of cytosolic $\boldsymbol{S}$. Infantis Caco-2 cells. A Observation of the apical surface of Caco-2 cell monolayer ultrastructure with scanning electron microscopy. SI(a), SI(b) and SI(c) showed that S. Infantis infected cell was bacteria-laden and these bacteria were extruded out of the monolayer. B Western blot analysis for the expression levels of claudin-1, occludin, and ZO-1 proteins. The right panel shows the quantitation analysis by Image J software $\left(n=3,{ }^{*} P<0.01,{ }^{* *} P<0.001\right)$. C Detection of the distribution and expression of ZO-1 (Green) in Caco-2 cells by immunofluorescence staining. Red: Salmonella. Blue: DAPI. Scale bar, $10 \mu \mathrm{m}$. D Calcein-AM/PI cell mortality test for S. Infantis-induced cell death rate. $\mathbf{E}$ Effect of butyrate on intracellular bacteria as assessed by the gentamicin protection assay. $\mathbf{F}$ The intracellular bacteria were assessed by the gentamicin protection assay, without (total intracellular bacteria, black curve) or with (cytosolic bacteria, gray curve) chloroquine. G The number of the infected cells containing 1 to 10, 10 to 20,20 to 50, or more than 50 bacteria per cell at 8 hpi was measured using a fluorescence microscope. At least 100 cells were counted for each group and the percentage of cells containing the different numbers of bacteria is shown (mean $\pm \mathrm{SEM}, \mathrm{n}=3$ ), ${ }^{* *} P<0.01,{ }^{* * *} P<0.001$. $\mathbf{H}$ Caco-2 cells were infected with $\mathrm{S}$. Infantis-pFPV (Green). Cells at 8 hpi were stained with LAMP-1 (Red) to label S. Infantis in SCV, while the cytosolic S. Infantis were green. "Zoom" denotes a magnified image of the white dotted area. Blue: DAPI. Scale bar, $10 \mu \mathrm{m}$.

the LC3-II/I ratio in the colon, but this increase was attenuated by treatment with the CBB-MIX (Figure 5A). Similarly, the increase in ATG5 and Beclin1 protein levels as well as the LC3-II/I ratio induced by $S$. Infantis at 8 hpi in Caco-2 cells was inhibited by butyrate (Figure 5B). In contrast, the protein level of p62 was upregulated both in vivo and in vitro (Figure 5A, B). An increased p62 protein level usually implies that the autophagy flux is blocked, especially at the stage of lysosome-mediated autophagic degradation [39]. We assumed that $S$. Infantis might disturb the function of lysosomes. Western blot assays showed that compared with control cells, $S$. Infantis infection downregulated the expression of the glycosylated membrane structural proteins LAMP1 and LAMP2, as well as ATP6V1A, ATP6V1B2, and ATP6V1E1. This decrease was alleviated by butyrate 


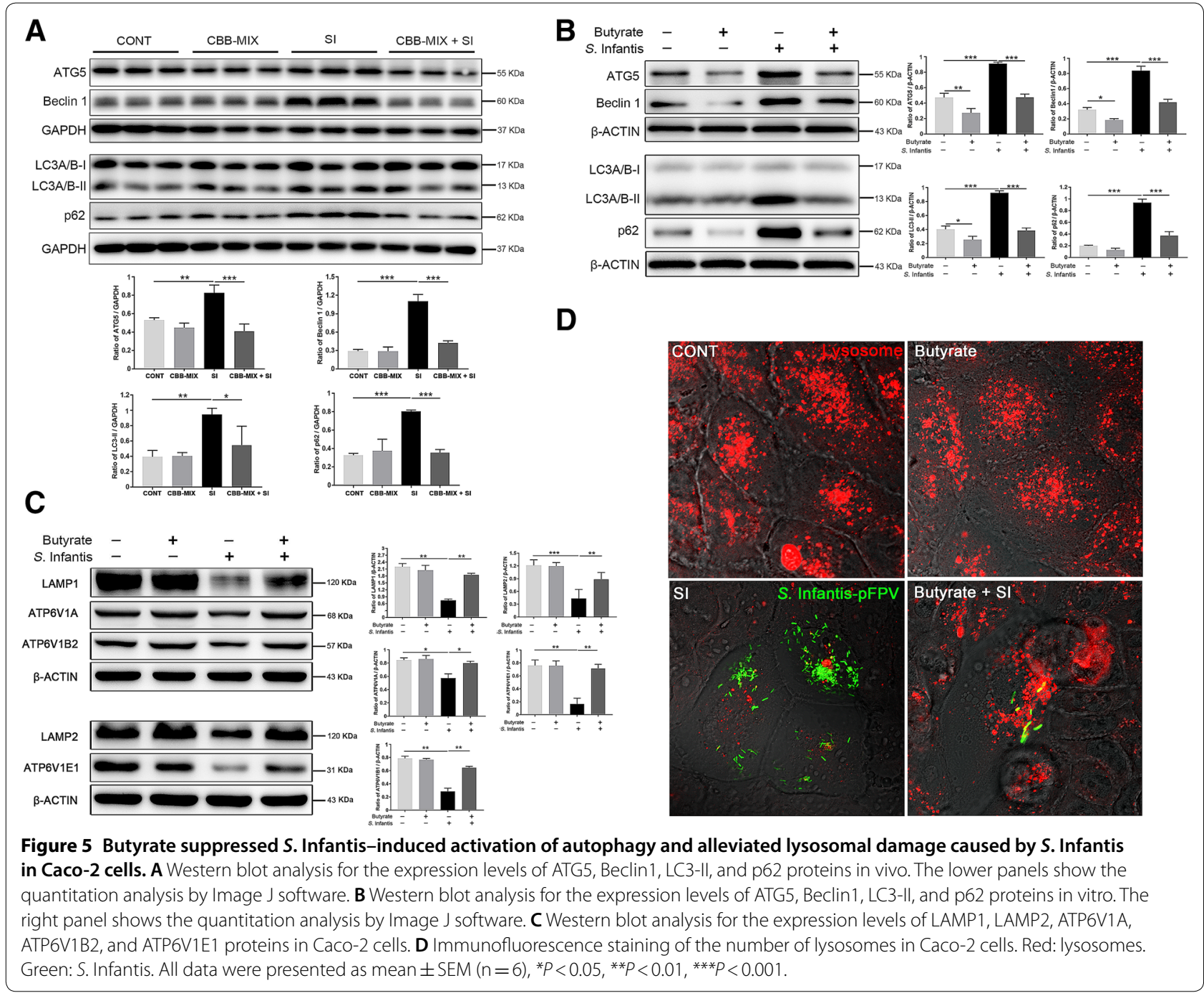

(Figure 5C). Furthermore, immunofluorescence images demonstrated that $S$. Infantis infection led to decreased numbers of lysosomes and these were smaller in size. Butyrate treatment prevented this situation (Figure 5D), suggesting that it suppressed $S$. Infantis-induced activation of autophagy and alleviated lysosomal damage caused by $S$. Infantis.

\section{Butyrate limited replication of cytosolic $S$. Infantis by inhibiting autophagy in Caco- 2 cells}

We found that butyrate limited the replication of cytosolic $S$. Infantis and inhibited autophagy in Caco-2 cells during $S$. Infantis infection, but the role of autophagy during $S$. Infantis infection is still unclear. One recent study showed that autophagy facilitated Salmonella replication in HeLa cells [19]. We assumed that butyrate restricted $S$. Infantis replication through suppressing autophagy. To verify this hypothesis, we depleted ATG5 levels by siRNA. Western blot assays confirmed that siRNA effectively suppressed the expression of ATG5 (Figure 6A). Knockdown of ATG5 decreased S. Infantisinduced autophagy, which was confirmed by the decrease of the LC3-II protein level (Figure 6B). Compared with the untreated cells, $S$. Infantis infection reduced the level of $\mathrm{ZO}-1$ protein at $8 \mathrm{hpi}$, but this reduction was reversed by knockdown of ATG5 (Figure 6B). Immunofluorescence analysis further confirmed that knockdown of ATG5 decreased the number of intracellular $S$. Infantis and ameliorated $S$. Infantis-induced disorder of ZO-1 protein arrangement and aggregation between cells (Figure 6C). Furthermore, ATG5 knockdown decreased the $S$. Infantis-induced cell mortality from 39.6 to $13.8 \%$ (Figure 6D).

We evaluated the effect of ATG5 knockdown on the replication of cytosolic $S$. Infantis. Similar to butyrate, ATG5 knockdown did not attenuate the invasiveness of 

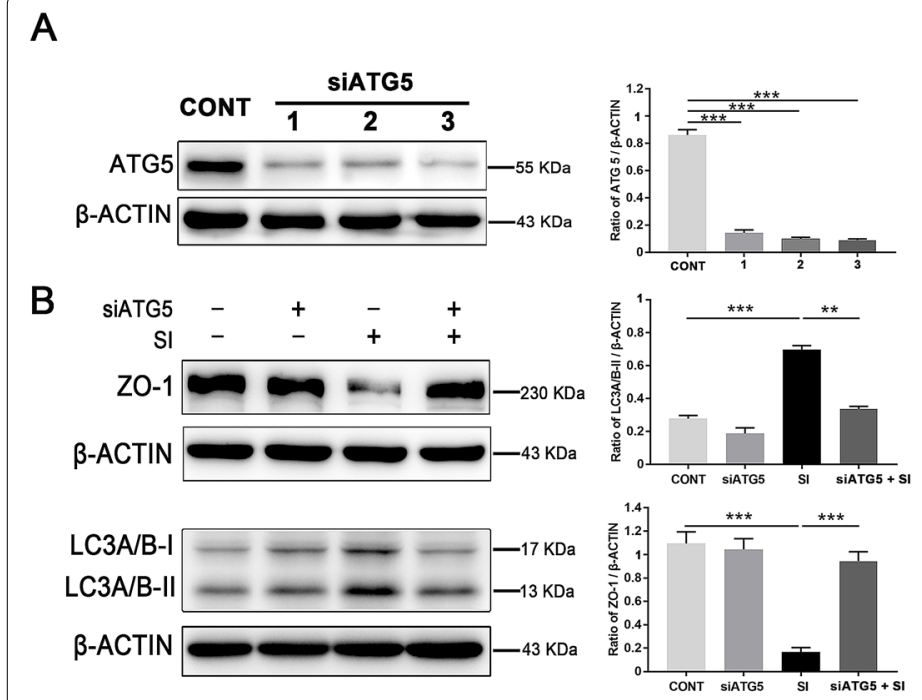
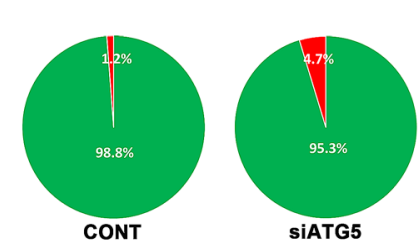

SiATG5

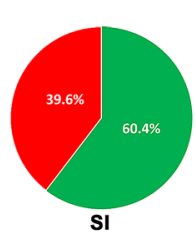

SI
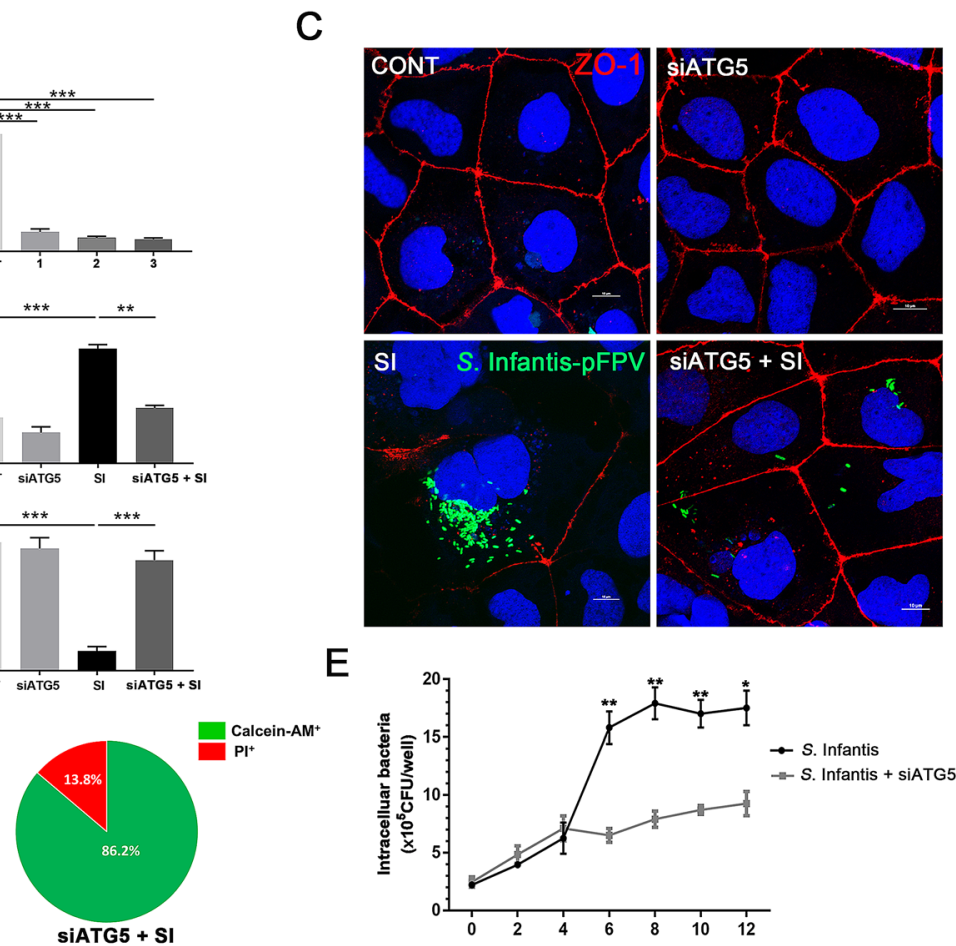

E

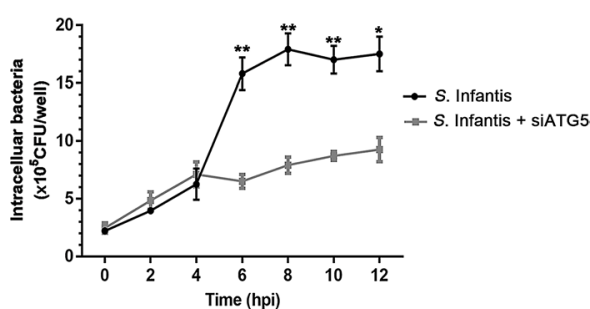

$\mathrm{F}$

G
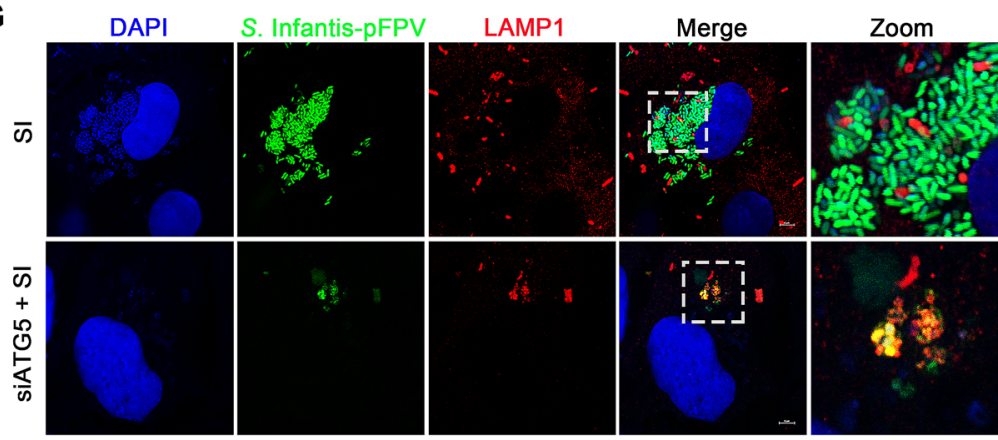

Figure 6 Butyrate limited replication of cytosolic S. Infantis by inhibiting autophagy. A Western blot analysis of Caco-2 cells treated with siRNA-targeting ATG5 (mean $\pm S E M, n=3$ ), ${ }^{* *} P<0.001$. B Western blot analysis for the expression levels of ZO-1 and LC3 proteins. The right panel shows the quantitation analysis by ImageJ software. (mean $\pm S E M, n=3$ ), ${ }^{* *} P<0.01,{ }^{* * *} P<0.001$. C Detection of the distribution and expression of ZO-1 (Red) of Caco-2 cells by immunofluorescence staining. Blue: DAPI, Scale bar, $10 \mu \mathrm{m}$. D Cell death was measured using Calcein-AM/PI assay. E Effect of siATG5 on intracellular S. Infantis was assessed by the gentamicin protection assay. F The number of infected cells containing 1 to 10, 10 to 20, 20 to 50, or more than 50 bacteria per cell at $8 \mathrm{hpi}$ was measured using a fluorescence microscope. At least 100 cells were counted for each group and the percentage of cells with the different numbers of bacteria is presented (mean $\pm S E M, n=3$ ), ${ }^{*} P<0.05,{ }^{* *} P<0.01$. G Caco-2 cells were infected with S. Infantis-pFPV. Cells at 8 hpi were stained with LAMP-1 (Red) to label S. Infantis in SCV, while the cytosolic S. Infantis was green. "Zoom" denotes a magnified image of the white dotted area. Green: S. Infantis. Blue: DAPI. Scale bar, $10 \mu \mathrm{m}$.

S. Infantis, as assessed by the intracellular bacteria CFU during the first $2 \mathrm{hpi}$, but restricted the replication of intracellular S. Infantis between 6 to 12 hpi (Figure 6E). The proportion of cells with bacterial hyper-replication also decreased from 30.1 to $1.3 \%$ (Figure 6F). Indeed, the proportion of cells infected with less than 20 bacteria per cell in the siATG5 + SI group was higher than that of cells infected with $S$. Infantis alone $(91.2 \%$ vs
39.5\%). Additionally, the quantity of LAMP1- bacteria (cytosolic) in the siATG5 + SI group markedly decreased compared with that in the SI group, while no visible differences were observed in the amount of LAMP1 ${ }^{+}$bacteria between the treatment groups (SCV) (Figure 6G). The results showed that butyrate limited the proliferation of cytosolic $S$. Infantis by inhibiting autophagy. 
Taken together, these results suggest that treatment with CBB-MIX maintained the abundance of butyrateproducing bacteria in the colon and restored the butyrate level in weaned pigs infected with $S$. Infantis. Butyrate reduced cell damage by inhibiting autophagy to limit replication of cytosolic $S$. Infantis.

\section{Discussion}

The CBB-MIX was composed of three strains: L. johnsonii CJ21, B. subtilis BS15, and B. licheniformis BL21, all of which were isolated from the intestinal contents of healthy pigs. Lactobacillus can improve the butyrate production capacity of butyrate producing bacteria in the intestinal tract [40]. Butyrate can activate the PPAR- $\gamma$ signaling pathway of intestinal epithelial cells. This, in turn drives the energy metabolism of colon cells to transform into mitochondrial $\beta$-oxidation using butyric acid as the energy supply, consuming large amounts of oxygen. This form of energy metabolism is very important for the consumption of oxygen in the intestine. Only the continuous and stable mitochondrial $\beta$-oxidation in intestinal epithelial cells can ensure the formation of a physiological anoxic intestinal environment, thus ensuring a healthy microbiota structure dominated by specialized anaerobic bacteria [41, 42]. Bacillus can consume large amounts of oxygen in the intestine and accelerate the generation of an anaerobic environment in the gut [43-45]. It was reported that cyclic dipeptides containing Val-Leu and Val-Ile produced by B. subtilis C-3102 could serve as bifidogenic growth factors to increase the abundance of beneficial microorganisms related to the genus Bifidobacterium in a human colonic microbiota model culture system [46]. B. subtilis PB6 supplementation reduced $S$. Typhimurium level in the transverse colon of weaned Holstein steers at $48 \mathrm{~h}$ after $S$. Typhimurium infection [47]. In our previous work, we found that oral administration of a selected mixture of $B$. licheniformis and B. subtilis probiotics can regulate the microbial community structure in the colon and protect weaned pigs against Enterotoxigenic E. coli infection [48]. In addition, our previous studies using a piglet model with $S$. Infantis infection showed that a mixture of Lactobacillus and Bacillus reduced the number of Salmonella in the feces of piglets, while L. johnsonii administration reduced the degree of Salmonella colonization and expansion in various intestinal segments [30, 49]. Furthermore, it was found that $B$. subtilis B2A supplementation reduced the intestinal Salmonella burden in broiler chicks [50,51].

In the present study, we found that the oral CBB-MIX treatment increased the abundance of butyrate-producing bacteria, including Faecalibacterium, Ruminococcaceae, Lachnospiraceae, and Blautia during the process of $S$. Infantis infection. These butyrate-producing bacteria can metabolize substrates to produce butyrate in the colon and most of them require an extremely strict anaerobic environment. Treatment with the CBB-MIX provided suitable conditions for the growth of these genera. Further, an elevated butyrate level was observed in the colon contents from the pigs pretreated with CBBMIX. Butyrate is the terminal product of fermentation of specific anaerobic microbiota in the intestine which specifically down-regulates Salmonella pathogenicity island 1 gene expression [52]. Addition of sodium butyrate was reported to decrease Salmonella seroprevalence in fattening pigs [53], while administration of butyrate induced antimicrobial activity in intestinal macrophages in vivo and increased resistance to enteropathogens [54]. Our data suggested that treatment with the CBB-MIX enriched butyrate-producing bacteria and increased butyrate levels in the colon contents, thereby providing defense against $S$. Infantis infection in pigs.

Notably, butyrate suppresses autophagy at physiological concentrations [29]. In the present study, we found that butyrate limited the proliferation of cytosolic $S$. Infantis by suppressing autophagy, which alleviated cell damage. This conclusion challenged the current concept that autophagy protects the host against Salmonella infection [55-57]. A recent study suggested that HilA, a transcriptional regulator of SPI-1, can be acylated by butyrate, which weakens Salmonella Typhimurium invasion into epithelial cells [58]. In this study, we specifically excluded this factor by removing butyrate from the culture medium during the first $30 \mathrm{~min}$ of $S$. Infantis infection. The intracellular bacterial load assessment also showed that butyrate did not attenuate the invasiveness of $S$. Infantis. Subsequently, we showed that suppression of autophagy by ATG5 knockdown did inhibit the proliferation of cytosolic $S$. Infantis. We speculate that the time point of quantifying bacteria in cells may be an important consideration, as some studies have demonstrated that the autophagy receptor protein p62 limits Salmonella replication at $10 \mathrm{hpi}$ [59]. Thus we quantified bacterial load at $8 \mathrm{hpi}$ to avoid negative results due to cell separation or death at late time points. Consistent with our view that inhibition of autophagy limited Salmonella replication. Huang found the proliferation of Salmonella was slowed down when Rab1 was depleted, an essential GTPase for autophagy [18]. What is more convincing is that Yu et al. demonstrated that p62-dependent canonical autophagy was beneficial for the replication of cytosolic Salmonella in HeLa cells [19]. They observed a subpopulation of cytosolic Salmonella associated extensively with p62 or LC3 and the replication rate was much faster than that in SCV with a living cell station. It has been proved that cytosolic replication of Salmonella contributed to the majority of Salmonella net replication $[8,11,38]$. 
These intuitive evidence showed that autophagy favors the replication of Salmonella. Additionally, the fate of Salmonella is different after invading different types of cells. For example, there is no "free" cytosolic Salmonella within fibroblasts due to the effective autophagy response [60]. In macrophages, reactive oxygen and nitrogen species with strong activity can effectively kill invading bacteria $[61,62]$. It is noteworthy that autophagy can also be "hijacked" by some other pathogens, like Legionella, Coxiella, and Yersinia [63]. Once fused with lysosomes to form autophagolysosomes, these organelles may serve as a source of nutrients for these intracellular pathogens. Autophagosomes may also act as a protective shelter for intracellular pathogens [64].

In conclusion, our data demonstrated that treatment with the CBB-MIX had strong properties in maintaining the composition and abundance of beneficial microbiota in the colon of piglets in the presence of $S$. Infantis, most especially the butyrate-producing bacteria. Butyrate is a metabolite of the beneficial microbiota, which can limit the proliferation of cytosolic $S$. Infantis in Caco-2 cells by inhibiting autophagy. The increasing situation of antibiotic resistance and emergence of new strains of $S$. Infantis indicate that a more thorough understanding of host-pathogen interactions and more novel antibiotic substitutes are urgently needed. Our research established a probiotic protection model based on a microbial metabolite, which provides a new perspective for better understanding of the protective activity of probiotics against pathogens.

\begin{abstract}
Abbreviations
ASC: apoptosis-associated speck-like protein containing a CARD; Atg: autophagy-related gene; B. subtilis: Bacillus subtilis; B. licheniformis: Bacillus licheniformis; Caco: colon adenocarcinoma cells; CFU: colony-forming unit; CQ: chloroquine; DAPI: 4',6'-Diamidino-2-phenylindole; hpi: hours post infection; LAMP: Iysosomal-associated membrane protein; L. johnsonii: Lactobacillus johnsonii; LC3: microtubule-binding protein light chain 3; PBS: phosphatebuffered saline; S. Infantis: Salmonella enterica serovar Infantis; SCFAs: short chain fatty acids; SCV: Salmonella-containing vacuole; SEM: scanning electron microscopy; SQSTM1: sequestosome 1; ZO-1: zona occludens protein 1.
\end{abstract}

\section{Acknowledgements}

We thank the following funds for their support in this work: the National Key R\&D Program of China (Project No. 2017YFD0502200), the National Natural Science Foundation of China (Project Nos. 31873034 and 31672613), and Beijing Natural Science Foundation (Project No. 6202006).

\section{Authors' contributions}

$B C$ designed and performed the majority of experiments and wrote the paper with input from all authors. YZ participated in experimental design and provided advice during the experiment procedure. JS and BX participated in the design of the animal experiments. JS, BX, YZ, and JN assisted with the experiments. WZ and JW are responsible for the funding collection and revising the paper. All authors read and approved the final manuscript.

\section{Funding}

This work was supported by the National Key R\&D Program of China (Project No. 2017YFD0502200), the National Natural Science Foundation of China
(Project Nos. 31873034 and 31672613), and Beijing Natural Science Foundation (Project No. 6202006).

\section{Availability of data and materials}

All data in this study are included in this article.

\section{Ethics approval and consent to participate}

All the experimental animals in this study were treated in strict accordance with the Guidelines for Laboratory Animal Use and Care from the Chinese Center for Disease Control and Prevention and the Rules for Medical Laboratory Animals (1998) from the Chinese Ministry of Health, under protocol CAU20151001-1, which was approved by the Animal Ethics Committee of the China Agricultural University. All animals were euthanized under pentobarbital sodium anesthesia and every effort was made to alleviate pain.

\section{Consent to publish}

The authors agrees to publication in Veterinary Research.

\section{Competing interests}

The authors declare that they have no competing interests.

Received: 3 April 2020 Accepted: 22 July 2020

Published online: 05 August 2020

\section{References}

1. Tate H, Folster JP, Hsu CH, Chen J, Hoffmann M, Li C, Morales C, Tyson GH, Mukherjee S, Brown AC, Green A, Wilson W, Dessai U, Abbott J, Joseph L, Haro J, Ayers S, McDermott PF, Zhao S (2017) Comparative analysis of extended spectrum- $\beta$-lactamase CTX-M-65-producing Salmonella enterica serovar Infantis isolates from humans, food animals, and retail chickens in the United States. Antimicrob Agents Ch 61:e00488-17

2. Borowiak M, Szabo I, Baumann B, Junker E, Hammerl JA, Kaesbohrer A, Malorny B, Fischer J (2017) VIM-1-producing Salmonella Infantis isolated from swine and minced pork meat in Germany. J Antimicrob Chemother 72:2131-2133

3. Aviv G, Tsyba K, Steck N, Salmon-Divon M, Cornelius A, Rahav G, Grassl GA, Gal-Mor O (2014) A unique megaplasmid contributes to stress tolerance and pathogenicity of an emergent Salmonella enterica serovar Infantis strain. Environ Microbiol 16:977-994

4. Aviv G, Elpers L, Mikhlin S, Cohen H, Vitman Zilber S, Grassl GA, Rahav G, Hensel M, Gal-Mor O (2017) The plasmid-encoded Ipf and Klf fimbriae display different expression and varying roles in the virulence of Salmonella enterica serovar Infantis in mouse vs. avian hosts. PLoS Pathog 13:e1006559

5. Schroeder S, Harries M, Prager R, Höfig A, Ahrens B, Hoffmann L, Rabsch W, Mertens E, Rimek D (2016) A prolonged outbreak of Salmonella Infantis associated with pork products in central Germany, April-October 2013. Epidemiol Infect 144:1429-1439

6. Braukmann M, Methner U, Berndt A (2015) Immune reaction and survivability of Salmonella typhimurium and Salmonella Infantis after infection of primary avian macrophages. PLoS One 10:e0122540

7. Steele-Mortimer O (2008) The Salmonella-containing vacuole: moving with the times. Curr Opin Microbiol 11:38-45

8. Malik-Kale P, Winfree S, Steele-Mortimer O (2012) The bimodal lifestyle of intracellular Salmonella in epithelial cells: replication in the cytosol obscures defects in vacuolar replication. PLoS One 7:e38732

9. Bujny MV, Ewels PA, Humphrey S, Attar N, Jepson MA, Cullen PJ (2008) Sorting nexin-1 defines an early phase of Salmonella-containing vacuoleremodeling during Salmonella infection. J Cell Sci 121:2027-2036

10. Bakowski MA, Braun V, Brumell JH (2008) Salmonella-containing vacuoles: directing traffic and nesting to grow. Traffic 9:2022-2031

11. Knodler LA (2015) Salmonella enterica: living a double life in epithelial cells. Curr Opin Microbiol 23:23-31

12. Knodler LA, Vallance BA, Celli J, Winfree S, Hansen B, Montero M, Steele-Mortimer O (2010) Dissemination of invasive Salmonella via bacterial-induced extrusion of mucosal epithelia. Proc Natl Acad Sci USA 107:17733-17738

13. Levine B, Kroemer G (2008) Autophagy in the pathogenesis of disease. Cell 132:27-42 
14. Mizushima N (2007) Autophagy: process and function. Gene Dev 21:2861-2873

15. Gomes LC, Dikic I (2014) Autophagy in antimicrobial immunity. Mol Cell 54:224-233

16. Bauckman KA, Owusu-Boaitey N, Mysorekar IU (2015) Selective autophagy: xenophagy. Methods 75:120-127

17. Wild P, Farhan H, McEwan DG, Wagner S, Rogov W, Brady NR, Richter B, Korac J, Waidmann O, Choudhary C, Dötsch V, Bumann D, Dikic I (2011) Phosphorylation of the autophagy receptor optineurin restricts Salmonella growth. Science 333:228-233

18. Huang J, Birmingham CL, Shahnazari S, Shiu J, Zheng YT, Smith AC, Campellone KG, Heo WD, Gruenheid S, Meyer T, Welch MD, Ktistakis NT, Kim PK, Klionsky DJ, Brumell JH (2011) Antibacterial autophagy occurs at $\mathrm{PI}(3) \mathrm{P}$-enriched domains of the endoplasmic reticulum and requires Rab1 GTPase. Autophagy 7:17-26

19. Yu HB, Croxen MA, Marchiando AM, Ferreira RB, Cadwell K, Foster LJ, Finlay BB (2014) Autophagy facilitates Salmonella replication in HeLa cells. mBio 5:e00865-00814

20. Siqueira MDS, Ribeiro RM, Travassos LH (2018) Autophagy and its interaction with intracellular bacterial pathogens. Front Immunol 9:935

21. Zhang W, Zhu YH, Yang GY, Liu X, Xia B, Hu X, Su JH, Wang JF (2017) Lactobacillus rhamnosus GG affects microbiota and suppresses autophagy in the intestines of pigs challenged with Salmonella Infantis. Front Microbiol 8:2705

22. Xia B, Yu J, He T, Liu X, Su JH, Wang MJ, Wang JF, Zhu YH (2020) Lactobacillus johnsonii L531 ameliorates enteritis via elimination of damaged mitochondria and suppression of SQSTM1-dependent mitophagy in a Salmonella Infantis model of pig diarrhea. FASEB J 34:2821-2839

23. Drumo R, Pesciaroli M, Ruggeri J, Tarantino M, Chirullo B, Pistoia C, Petrucci P, Martinelli N, Moscati L, Manuali E, Pavone S, Picciolini M, Ammendola S, Gabai G, Battistoni A, Pezzotti G, Alborali GL, Napolioni V, Pasquali P, Magistrali CF (2016) Salmonella enterica serovar Typhimurium exploits inflammation to modify swine intestinal microbiota. Front Cell Infect Microbiol 5:106

24. Shavakhi A, Tabesh E, Yaghoutkar A, Hashemi H, Tabesh F, Khodadoostan M, Minakari M, Shavakhi S, Gholamrezaei A (2013) The effects of multistrain probiotic compound on bismuth-containing quadruple therapy for Helicobacter pylori infection: a randomized placebo-controlled tripleblind study. Helicobacter 18:280-284

25. Ekmekciu I, von Klitzing E, Fiebiger U, Neumann C, Bacher P, Scheffold A, Bereswill S, Heimesaat MM (2017) The probiotic compound VSL\# 3 modulates mucosal, peripheral, and systemic immunity following murine broad-spectrum antibiotic treatment. Front Cell Infect Microbiol 7:167

26. Tan J, McKenzie C, Potamitis M, Thorburn AN, Mackay CR, Macia L (2014) The role of short-chain fatty acids in health and disease. Adv Immunol 121:91-119

27. Macfarlane GT, Sandra M (2012) Bacteria, colonic fermentation, and gastrointestinal health. J AOAC Int 95:50-60

28. Bourassa MW, Alim I, Bultman SJ, Ratan RR (2016) Butyrate, neuroepigenetics and the gut microbiome: can a high fiber diet improve brain health? Neurosci Lett 625:56-63

29. Donohoe DR, Garge N, Zhang X, Sun W, O'Connell TM, Bunger MK, Bultman SJ (2011) The microbiome and butyrate regulate energy metabolism and autophagy in the mammalian colon. Cell Metab 13:517-526

30. He T, Zhu YH, Yu J, Xia B, Liu X, Yang GY, Su JH, Guo L, Wang ML, Wang JF (2019) Lactobacillus johnsonii L531 reduces pathogen load and helps maintain short-chain fatty acid levels in the intestines of pigs challenged with Salmonella enterica Infantis. Vet Microbiol 230:187-194

31. Yang GY, Yu J, Su JH, Jiao LG, Liu X, Zhu YH (2017) Oral administration of Lactobacillus rhamnosus GG ameliorates Salmonella Infantis-induced inflammation in a pig model via activation of the IL-22BP/IL-22/STAT3 pathway. Front Cell Infect Microbiol 7:323

32. Zhou D, Zhu YH, Zhang W, Wang ML, Fan WY, Song D, Yang GY, Jensen BB, Wang JF (2015) Oral administration of a select mixture of Bacillus probiotics generates $\operatorname{Tr} 1$ cells in weaned $\mathrm{F} 4 \mathrm{ab} / \mathrm{acR} \mathrm{R}^{-}$pigs challenged with an $\mathrm{F}^{+}$ ETECNTEC/EPEC strain. Vet Res 46:95

33. Shen YB, Piao XS, Kim SW, Wang L, Liu P, Yoon I, Zhen YG (2009) Effects of yeast culture supplementation on growth performance, intestinal health, and immune response of nursery pigs. J Anim Sci 87:2614-2624

34. Wu Q, Liu MC, Yang J, Wang JF, Zhu YH (2016) Lactobacillus rhamnosus GR-1 ameliorates Escherichia coli-induced inflammation and cell damage via attenuation of ASC-independent NLRP3 inflammasome activation. Appl Environ Microbiol 82:1173-1182

35. Turner JR (2009) Intestinal mucosal barrier function in health and disease Nat Rev Immunol 9:799-809

36. Yang CM, Ferket PR, Hong QH, Zhou J, Cao GT, Zhou L, Chen AG (2012) Effect of chito-oligosaccharide on growth performance, intestinal barrier function, intestinal morphology and cecal microflora in weaned pigs. J Anim Sci 90:2671-2676

37. Castanheira S, Garcia-Del Portillo F (2017) Salmonella populations inside host cells. Front Cell Infect Microbiol 7:432

38. Knodler LA, Nair V, Steele-Mortimer O (2014) Quantitative assessment of cytosolic Salmonella in epithelial cells. PLoS One 9:e84681

39. Chu BX, Fan RF, Lin SQ, Yang DB, Wang ZY, Wang L (2018) Interplay between autophagy and apoptosis in lead(II)-induced cytotoxicity of primary rat proximal tubular cells. J Inorg Biochem 182:184-193

40. Lin R, Sun Y, Mu P, Zheng T, Wen J (2020) Lactobacillus rhamnosus GG supplementation modulates the gut microbiota to promote butyrate production, protecting against deoxynivalenol exposure in nude mice. Biochem Pharmacol 175:113868

41. Patrice DC (2017) Gut cell metabolism shapes the microbiome. Science 357:548-549

42. Byndloss MX, Olsan EE, Rivera-Chavez F, Tiffany CR, Cevallos SA, Lokken KL, Torres TP, Byndloss AJ, Faber F, Gao Y, Litvak Y, Lopez CA, Xu G, Napoli E, Giulivi C, Tsolis RM, Revzin A, Lebrilla CB, Baumler AJ (2017) Microbiotaactivated PPAR- $\gamma$ signaling inhibits dysbiotic enterobacteriaceae expansion. Science 357:570-575

43. Sun $Y Z$, Yang HL, Ma RL, Lin WY (2010) Probiotic applications of two dominant gut Bacillus strains with antagonistic activity improved the growth performance and immune responses of grouper Epinephelus coioides. Fish Shellfish Immunol 29:803-809

44. Deng W, Dong XF, Tong JM, Zhang Q (2012) The probiotic Bacillus licheniformis ameliorates heat stress-induced impairment of egg production, gut morphology, and intestinal mucosal immunity in laying hens. Poult Sci 91:575-582

45. Cui C, Shen CJ, Jia G, Wang KN (2013) Effect of dietary Bacillus subtilis on proportion of Bacteroidetes and Firmicutes in swine intestine and lipid metabolism. Genet Mol Res 12:1766-1776

46. Hatanaka M, Morita H, Aoyagi Y, Sasaki K, Sasaki D, Kondo A, Nakamura T (2020) Effective bifidogenic growth factors cyclo-Val-Leu and cyclo-Vallle produced by Bacillus subtilis C-3102 in the human colonic microbiota model. Sci Rep 10:7591

47. Broadway PR, Carroll JA, Sanchez NCB, Callaway TR, Lawhon SD, Gart EV, Bryan LK, Nisbet DJ, Hughes HD, Legako JF, Hergenreder JE, Rounds PW (2020) Bacillus subtilis pb6 supplementation in weaned holstein steers during an experimental Salmonella challenge. Foodborne Pathog Dis $17: 8$

48. Zhang W, Zhu YH, Zhou D, Wu Q, Song D, Dicksved J, Wang JF (2017) Oral administration of a select mixture of Bacillus probiotics affects the gut microbiota and goblet cell function following Escherichia coli challenge in newly weaned pigs of genotype MUC4 that are supposed to be enterotoxigenic E. coli F4ab/ac receptor negative. Appl Environ Microbiol 83:e02747-16

49. Liu X, Xia B, He T, Li D, Su JH, Guo L, Wang JF, Zhu YH (2019) Oral administration of a select mixture of Lactobacillus and Bacillus alleviates inflammation and maintains mucosal barrier integrity in the ileum of pigs challenged with Salmonella Infantis. Microorganisms 7:135

50. Park JH, Kim IH (2014) Supplemental effect of probiotic Bacillus Subtilis b2a on productivity, organ weight, intestinal Salmonella microflora, and breast meat quality of growing broiler chicks. Poultry Sci 93:2054-2059

51. La Ragione RM (2003) Competitive exclusion by Bacillus Subtilis spores of Salmonella enterica serotype enteritidis and Clostridium perfringens in young chickens. Vet Microbiol 94:245-256

52. Gantois I, Ducatelle R, Pasmans F, Haesebrouck F, Hautefort I, Thompson A, Hinton JC, Van Immerseel F (2006) Butyrate specifically down-regulates Salmonella pathogenicity island 1 gene expression. Appl Environ Microbiol 72:946-949

53. Casanova-Higes A, Andrés-Barranco S, Mainar-Jaime RC (2017) Effect of the addition of protected sodium butyrate to the feed on Salmonella spp. infection dynamics in fattening pigs. Anim Feed Sci Tech 231:12-18

54. Schulthess J, Pandey S, Capitani M, Rue-Albrecht KC, Arnold I, Franchini F, Chomka A, Ilott NE, Johnston DGW, Pires E, McCullagh J, Sansom SN, 
Arancibia-Cárcamo CV, Uhlig HH, Powrie F (2019) The short chain fatty acid butyrate imprints an antimicrobial program in macrophages. Immunity 50:432-445

55. Birmingham CL, Smith AC, Bakowski MA, Yoshimori T, Brumell JH (2006) Autophagy controls Salmonella infection in response to damage to the Salmonella-containing vacuole. J Biol Chem 281:11374-11383

56. Beuzón CR, Méresse S, Unsworth KE, Ruíz-Albert J, Garvis S, Waterman SR, Ryder TA, Boucrot E, Holden DW (2000) Salmonella maintains the integrity of its intracellular vacuole through the action of SifA. EMBO J 19:3235-3249

57. Casanova JE (2017) Bacterial autophagy: offense and defense at the hostpathogen interface. Cell Mol Gastroenterol Hepatol 4:237-243

58. Zhang ZJ, Pedicord VA, Peng T, Hang HC (2020) Site-specific acylation of a bacterial virulence regulator attenuates infection. Nat Chem Biol 16:95-103

59. Zheng YT, Shahnazari S, Brech A, Lamark T, Johansen T, Brumell JH (2009) The adaptor protein p62/SQSTM1 targets invading bacteria to the autophagy pathway. J Immunol 183:5909-5916

60. López-Montero N, Ramos-Marquès E, Risco C, García-Del Portillo F (2016) Intracellular Salmonella induces aggrephagy of host endomembranes in persistent infections. Autophagy 12:1886-1901
61. Perrin AJ, Jiang X, Birmingham CL, So NS, Brumell JH (2004) Recognition of bacteria in the cytosol of mammalian cells by the ubiquitin system. Curr Biol 14:806-811

62. Thurston TL, Matthews SA, Jennings E, Alix E, Shao F, Shenoy AR, Birrell MA, Holden DW (2016) Growth inhibition of cytosolic Salmonella by caspase-1 and caspase-11 precedes host cell death. Nat Commun 7:1-15

63. Mostowy S, Cossart P (2012) Bacterial autophagy: restriction or promotion of bacterial replication? Trends Cell Biol 22:283-291

64. Levine B, Mizushima N, Virgin HW (2011) Autophagy in immunity and inflammation. Nature 469:323-335

\section{Publisher's Note}

Springer Nature remains neutral with regard to jurisdictional claims in published maps and institutional affiliations.
Ready to submit your research? Choose BMC and benefit from:

- fast, convenient online submission

- thorough peer review by experienced researchers in your field

- rapid publication on acceptance

- support for research data, including large and complex data types

- gold Open Access which fosters wider collaboration and increased citations

- maximum visibility for your research: over 100M website views per year

At BMC, research is always in progress.

Learn more biomedcentral.com/submissions 